ISSN $1974-4110$

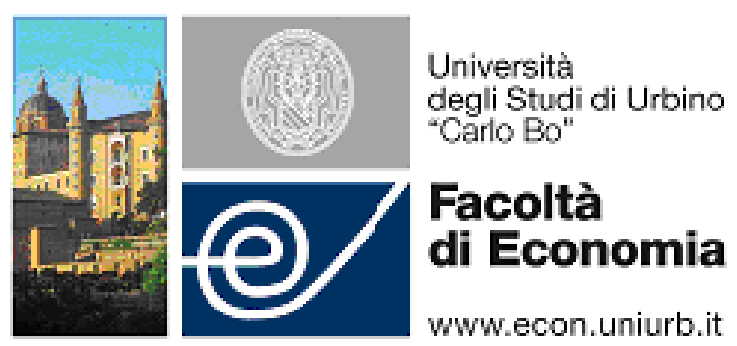

WP-EMS

Working Papers Series in Economics, Mathematics and Statistics

\begin{abstract}
"Generalized Hukuhara Differentiability of Interval-valued Functions and Interval Differential Equations"
\end{abstract}

- Barnabàs BEDE, (Univ. Texas-Pan American)

- Luciano STEFANINI, (Univ. Urbino) 



\title{
Generalized Hukuhara Differentiability of Interval-valued Functions and Interval Differential Equations
}

\author{
Luciano Stefanini, Barnabás Bede* \\ Faculty of Economics, University of Urbino Carlo Bo, Italy \\ Department of Mathematics, University of Texas-Pan American, Edinburg, Texas, \\ 78541 \\ Email adresses: lucste@uniurb.it, bedeb@utpa.edu
}

\begin{abstract}
In the present paper we introduce and study a generalization of the Hukuhara difference and also generalizations of the Hukuhara differentiability to the case of interval valued functions. We consider several possible definitions for the derivative of an interval valued function and we study connections between them and their properties. Using these concepts we study interval differential equations. Local existence and uniqueness of two solutions is obtained together with characterizations of the solutions of an interval differential equation by ODE systems and by differential algebraic equations. We also show some connection with differential inclusions. The thoretical results are turned into practical algorithms to solve interval differential equations.
\end{abstract}

Key words: interval-valued functions, generalized Hukuhara differentiability, interval differential equations

\section{Introduction}

Interval Analysis was introduced as an attempt to handle interval (non statistical, non probabilistic) uncertainty that appears in many mathematical or computer models of some deterministic real-world phenomena. The first monograph dealing with interval analysis is the celebrated book of R. Moore, [27]. Since then, Reliable Computing, Validated Numerics and Interval problems

\footnotetext{
* Corresponding author

Email address: bedeb@utpa.edu (Barnabás Bede).
} 
with Differential Equations are discussed in several monographs and research papers ([30], [28], [25], [29], [16], [26], [37], [17], [4]).

Another major approach to a set of similar problems is that of differential inclusions and multivalued analysis ([3], [12], [15], [22], [36]). This approach is also able to deal with discontinuous dynamical systems which do not fully fit into the Interval Analysis topic.

In classical Real Analysis, maybe one of the most important concepts is that of the derivative of a real-valued function. Correspondingly, in Interval Analysis or in the Theory of Differential Inclusions, we would expect to have a notion of the derivative of an interval-valued or set-valued function. Instead, the classical derivatives are used in both research directions which we have mentioned above. The reason for this is that a derivative concept which is both theoretically well founded and it is also applicable to concrete situations is still missing, despite the almost half a century of (otherwise very important) development of these domains.

Hukuhara derivative of a set-valued mapping was first introduced by Hukuhara in [19] and it has been studied in several works. The paper of Hukuhara was the starting point for the topic of Set Differential Equations and later also for Fuzzy Differential Equations. Recently, several works as e.g., [8], [23], [9], [24], [1], have brought back into the attention of the nonlinear analysis community, the topics of set differential equations and the Hukuhara derivative. Also, as a very important generalization and development related to the subject of the present paper is in the field of fuzzy sets, i.e., fuzzy calculus and fuzzy differential equations ([43], [33], [20], [35], [13], [18], [39], [41], [31], [34]).

Hukuhara's differentiability concept has an important drawback, that is the paradoxical behavior of the solutions of a set or a fuzzy differential equation, i.e., "irreversibility under uncertainty". This comes from the fact that a set (or fuzzy) differential equation may have only solutions with increasing length of their support, and the uncertainty is increasing as time goes by. So, however interval differential equations (IDE) are a natural way to model epistemic uncertainty of a dynamical system, they are not yet well understood because of the above mentioned drawback of Hukuhara's concept. Differential inclusions constitute one way to address the irreversibility problem of interval differential equations, however the numerical work in differential inclusions is even now not very well understood since in this case a derivative concept is missing.

Our point is that the generalization of the concept of Hukuhara differentiability can be of a great help in the study of interval differential equations (IDEs).

The idea of the presented approach and differentiability concept comes from a generalization of the Hukuhara difference for compact convex sets ( $\mathrm{gH}$ - 
difference) presented in [40] and the strongly and weakly generalized (Hukuhara) differentiability concepts proposed in [6]. Combining these notions we obtain very simple formulations of the concepts and results with weakly generalized Hukuhara derivative (gH-derivative) by the help of the concept of $\mathrm{gH}-$ difference. Let us also mention that this concept has a very intuitive interpretation too. The presented derivative concept is slightly more general than the notion of strongly generalized (Hukuhara) differentiability for the case of interval valued functions.

Also, our approach to interval differential equations is different from the approaches based on differential inclusions or interval analysis; we will see and discuss here that there is some connection among these approaches and the presented one and some connections with differential inclusions.

We prove several properties of the derivative concept considered here and also, we obtain characterization theorems for interval differential equation by ODEs. Namely, we show that any general interval (initial value) differential equation can be formulated, via gH-derivative, in terms of two systems of ODEs similarly to the results in [5]. Also we present a characterization result of IDEs via a particular (index-1 semi implicit) differential-algebraic equation (DAE) where the algebraic equation has two possible solutions, so producing a family of solutions to the DAE.

A supplementary motivation of this paper is that we would like to spread the above presented ideas in the communities dealing with interval analysis, multivalued functions and differential inclusions. Also, we would like to show that our results can be converted into practical algorithms, beyond the theoretical development of the topic.

In Section 2 we introduce a generalization of the Hukuhara difference which is used in Section 3 for defining a generalization of the Hukuhara derivative for the case of interval valued functions equivalent to a particularization of the weakly generalized (Hukuhara) differentiability proposed in [6] to the interval valued case. In Section 4 some results on integration of interval-valued functions are presented. Section 5 is dedicated to the study of interval differential equations. Here we show an existence result and we find connections between IDEs and ODEs and later, in Sect. 6 we find a connection between IDEs and DAEs. In Section 7 we provide some numerical algorithms to solve interval differential equations and we end up with examples and some conclusions. 


\section{Generalized Hukuhara difference}

Consider the space $\mathbb{R}^{n}$ of $n$-dimensional real numbers and let $\mathcal{K}_{C}^{n}$ be the space of nonempty compact and convex sets of $\mathbb{R}^{n}$. If $n=1$ denote $\mathbb{I}$ the set of (closed bounded) intervals of the real line. Given two elements $A, B \in \mathcal{K}_{C}^{n}$ and $k \in \mathbb{R}$, the usual interval arithmetic operations, i.e. Minkowski addition and scalar multiplication, are defined by $A+B=\{a+b \mid a \in A, b \in B\}$ and $k A=$ $\{k a \mid a \in A\}$. It is well known that addition is associative and commutative and with neutral element $\{0\}$. If $k=-1$, scalar multiplication gives the opposite $-A=(-1) A=\{-a \mid a \in A\}$ but, in general, $A+(-A) \neq\{0\}$, i.e. the opposite of $A$ is not the inverse of $A$ in Minkowski addition (unless $A=\{a\}$ is a singleton). A first implication of this fact is that, in general, additive simplification is not valid, i.e. $(A+C=B+C) \nRightarrow A=B$ or $(A+B)-B \neq A$ (the Minkowski difference is $A-B=A+(-1) B$ ).

To partially overcome this situation, the Hukuhara H-difference has been introduced as a set $C$ for which $A \ominus B=C \Longleftrightarrow A=B+C$ and an important property of $\Theta$ is that $A \ominus A=\{0\} \forall A \in \mathcal{K}_{C}^{n}$ and $(A+B) \ominus B=A, \forall A, B \in \mathcal{K}_{C}^{n}$.

The H-difference is unique, but it does not always exist (a necessary condition for $A \ominus B$ to exist is that $A$ contains a translate $\{c\}+B$ of $B)$.

A generalization of the Hukuhara difference proposed in [40] aims to partially overcome this situation.

Definition 1. ([40])The generalized Hukuhara difference of two sets $A, B \in$ $\mathcal{K}_{C}^{n}$ (gH-difference for short) is defined as follows

$$
A \ominus_{g} B=C \Longleftrightarrow\left\{\begin{array}{c}
(a) A=B+C \\
\text { or }(b) B=A+(-1) C
\end{array}\right.
$$

Remark 2. It is possible that $A=B+C$ and $B=A+(-1) C$ hold simultaneously; in this case, $A$ and $B$ translate into each other and $C$ is a singleton. In fact, $A=B+C$ implies $B+\{c\} \subseteq A \forall c \in C$ and $B=A+(-1) C$ implies $A-\{c\} \subseteq B \forall c \in C$ i.e. $A \subseteq B+\{c\}$; it follows that $A=B+\{c\}$ and $B=A+\{-c\}$. On the other hand, if $c^{\prime}, c^{\prime \prime} \in C$ then $A=B+\left\{c^{\prime}\right\}=B+\left\{c^{\prime \prime}\right\}$ and this requires $c^{\prime}=c^{\prime \prime}$.

The following properties were obtained in [40].

Proposition 3. Let $A, B \in \mathcal{K}_{C}^{n}$ be two compact convex sets; then

i) if the gH-difference exists, it is unique and it is a generalization of the usual Hukuhara difference since $A \ominus_{g} B=A \odot B$, whenever $A \odot B$ exists, 
ii) $A \ominus_{g} A=\{0\}$,

iii) if $A \ominus_{g} B$ exists in the sense (a), then $B \ominus_{g} A$ exists in the sense (b) and viceversa,

iv) $(A+B) \ominus_{g} B=A$,

v) $\{0\} \ominus_{g}\left(A \Theta_{g} B\right)=(-B) \Theta_{g}(-A)$,

vi) we have $\left(A \ominus_{g} B\right)=\left(B \ominus_{g} A\right)=C$ if and only if $C=\{0\}$ and $A=B$ (Note that, in general, $B-A=A-B$ with the Minkowski operations does not imply $A=B$ ).

In the unidimensional case (with $\mathcal{K}_{C}^{1}=\mathbb{I}$ ) the gH-difference exists for any two compact intervals. If $A=\left[a^{-}, a^{+}\right] \in \mathbb{I}$, we will denote by $\operatorname{len}(A)=a^{+}-a^{-}$ the length (or diameter) of interval $A$.

Proposition 4. The gH-difference of two intervals $A=\left[a^{-}, a^{+}\right]$and $B=$ $\left[b^{-}, b^{+}\right]$always exists and

$$
\begin{aligned}
{\left[a^{-}, a^{+}\right] \ominus_{g}\left[b^{-}, b^{+}\right] } & =\left[c^{-}, c^{+}\right] \\
\text {with } & \\
c^{-} & =\min \left\{a^{-}-b^{-}, a^{+}-b^{+}\right\} \\
c^{+} & =\max \left\{a^{-}-b^{-}, a^{+}-b^{+}\right\} .
\end{aligned}
$$

Conditions (a) and (b) in (1) are satisfied simultaneously if and only if the two intervals have the same length and $c^{-}=c^{+}$.

The metric structure is given usually by the Hausdorff distance between intervals: $D: \mathbb{I} \times \mathbb{I} \rightarrow \mathbb{R}_{+} \cup\{0\}$ with $D(A, B)=\max \left\{\left|a^{-}-b^{-}\right|,\left|a^{+}-b^{+}\right|\right\}$, where $A=\left[a^{-}, a^{+}\right]$and $B=\left[b^{-}, b^{+}\right]$. The following properties are well-known:

$$
\begin{aligned}
D(k A, k B) & =|k| D(A, B), \forall k \in \mathbb{R}, \\
D(A+C, B+C) & =D(A, B), \\
D(A+B, C+D) & \leq D(A, C)+D(B, D)
\end{aligned}
$$

and $(\mathbb{I}, D)$ is a complete and separable metric space.

An immediate property of the $\mathrm{gH}$-difference for $A, B \in \mathbb{I}$ is

$$
D(A, B)=0 \Longleftrightarrow A \ominus_{g} B=\{0\} .
$$

Limits and continuity can be characterized, in the metric $D$ for intervals, by the gH-difference. 
Proposition 5. Let $f:[a, b] \longrightarrow \mathbb{I}$ be such that $f(x)=\left[f^{-}(x), f^{+}(x)\right]$. Then we have

$$
\begin{aligned}
& \lim _{x \rightarrow x_{0}} f(x)=l \Longleftrightarrow \lim _{x \rightarrow x_{0}}\left(f(x) \ominus_{g} l\right)=\{0\} \\
& \lim _{x \rightarrow x_{0}} f(x)=f\left(x_{0}\right) \Longleftrightarrow \lim _{x \rightarrow x_{0}}\left(f(x) \ominus_{g} f\left(x_{0}\right)\right)=\{0\},
\end{aligned}
$$

where the limits are in the metric $D$ for intervals.

\section{Differentiation of interval valued functions}

In the followings we present alternative definitions for the derivative of an interval-valued function. Later we will prove that three of them are equivalent while one of the proposed differentiabilities is stronger than the other three concepts.

The first two concepts are particularizations of the fuzzy concepts presented in [6] to the interval case. These are using the usual Hukuhara difference " $\ominus$ ".

Definition 6. ([6]) Let $f:] a, b\left[\rightarrow \mathbb{I}\right.$ and $\left.x_{0} \in\right] a, b[$. We say that $f$ is strongly generalized (Hukuhara) differentiable at $x_{0}$, if there exists an element $f^{\prime}\left(x_{0}\right) \in$ $\mathbb{I}$, such that

(i) for all $h>0$ sufficiently small, $\exists f\left(x_{0}+h\right) \ominus f\left(x_{0}\right), f\left(x_{0}\right) \ominus f\left(x_{0}-h\right)$ and the limits

$$
\lim _{h \searrow 0} \frac{f\left(x_{0}+h\right) \ominus f\left(x_{0}\right)}{h}=\lim _{h \searrow 0} \frac{f\left(x_{0}\right) \ominus f\left(x_{0}-h\right)}{h}=f^{\prime}\left(x_{0}\right),
$$

or

(ii) for all $h>0$ sufficiently small, $\exists f\left(x_{0}\right) \ominus f\left(x_{0}+h\right), f\left(x_{0}-h\right) \ominus f\left(x_{0}\right)$ and the limits

$$
\lim _{h \searrow 0} \frac{f\left(x_{0}\right) \ominus f\left(x_{0}+h\right)}{(-h)}=\lim _{h \searrow 0} \frac{f\left(x_{0}-h\right) \ominus f\left(x_{0}\right)}{(-h)}=f^{\prime}\left(x_{0}\right),
$$

or

(iii) for all $h>0$ sufficiently small, $\exists f\left(x_{0}+h\right) \ominus f\left(x_{0}\right), f\left(x_{0}-h\right) \ominus f\left(x_{0}\right)$ and the limits

$$
\lim _{h \searrow 0} \frac{f\left(x_{0}+h\right) \ominus f\left(x_{0}\right)}{h}=\lim _{h \searrow 0} \frac{f\left(x_{0}-h\right) \ominus f\left(x_{0}\right)}{(-h)}=f^{\prime}\left(x_{0}\right),
$$

or 
(iv) for all $h>0$ sufficiently small, $\exists f\left(x_{0}\right) \ominus f\left(x_{0}+h\right), f\left(x_{0}\right) \ominus f\left(x_{0}-h\right)$ and the limits

$$
\lim _{h \searrow 0} \frac{f\left(x_{0}\right) \ominus f\left(x_{0}+h\right)}{(-h)}=\lim _{h \searrow 0} \frac{f\left(x_{0}\right) \ominus f\left(x_{0}-h\right)}{h}=f^{\prime}\left(x_{0}\right) .
$$

Definition 7. ([6])Let $f:] a, b\left[\rightarrow \mathbb{I}\right.$ and $\left.x_{0} \in\right] a, b\left[\right.$. For a sequence $h_{n} \searrow 0$ and $n_{0} \in \mathbb{N}$, let us denote

$$
\begin{aligned}
& A_{n_{0}}^{(1)}=\left\{n \geq n_{0} ; \exists E_{n}^{(1)}:=f\left(x_{0}+h_{n}\right) \ominus f\left(x_{0}\right)\right\}, \\
& A_{n_{0}}^{(2)}=\left\{n \geq n_{0} ; \exists E_{n}^{(2)}:=f\left(x_{0}\right) \ominus f\left(x_{0}+h_{n}\right)\right\}, \\
& A_{n_{0}}^{(3)}=\left\{n \geq n_{0} ; \exists E_{n}^{(3)}:=f\left(x_{0}\right) \ominus f\left(x_{0}-h_{n}\right)\right\}, \\
& A_{n_{0}}^{(4)}=\left\{n \geq n_{0} ; \exists E_{n}^{(4)}:=f\left(x_{0}-h_{n}\right) \ominus f\left(x_{0}\right)\right\} .
\end{aligned}
$$

We say that $f$ is weakly generalized (Hukuhara) differentiable on $x_{0}$, if for any sequence $h_{n} \searrow 0$, there exists $n_{0} \in \mathbb{N}$, such that $A_{n_{0}}^{(1)} \cup A_{n_{0}}^{(2)} \cup A_{n_{0}}^{(3)} \cup A_{n_{0}}^{(4)}=$ $\left\{n \in \mathbb{N} ; n \geq n_{0}\right\}$ and moreover, there exists an element in $\mathbb{I}$ denoted by $f^{\prime}\left(x_{0}\right)$, such that if for some $j \in\{1,2,3,4\}$ we have $\operatorname{card}\left(A_{n_{0}}^{(j)}\right)=+\infty$, then

$$
\lim _{\substack{n \rightarrow \infty \\ n \in A_{n_{0}}^{(j)}}} D\left(\frac{E_{n}^{(j)}}{(-1)^{j+1} h_{n}}, f^{\prime}\left(x_{0}\right)\right)=0 .
$$

Based on the gH-difference we propose the following

Definition 8. Let $\left.x_{0} \in\right] a, b\left[\right.$ and $h$ be such that $\left.x_{0}+h \in\right] a, b[$, then the gHderivative of a function $f:] a, b[\rightarrow \mathbb{I}$ can be defined as

$$
f^{\prime}\left(x_{0}\right)=\lim _{h \rightarrow 0} \frac{1}{h}\left[f\left(x_{0}+h\right) \ominus_{g} f\left(x_{0}\right)\right] .
$$

If $f^{\prime}\left(x_{0}\right) \in \mathbb{I}$ satisfying (2) exists, we say that $\mathrm{f}$ is generalized Hukuhara differentiable ( $g H$-differentiable for short) at $x_{0}$.

Remark 9. The gH-difference $f\left(x_{0}+h\right) \ominus_{g} f\left(x_{0}\right)$ always exists and for gHdifferentiability like above, $\operatorname{len}(f(x))$ is not necessarily increasing at $x_{0}$.

Remark 10. It is easy to see that the gH-differentiability concept introduced above is more general than the strongly generalized (Hukuhara) differentiability in Definition 6 (GH-differentiability for short). Case (i) of the GHdifferentiability (in fact the classical Hukuhara differentiability) corresponds to the existence of the gH-differences in case (a) over an interval $h \in] 0, \delta[$, $\delta>0$. Case (ii) of GH-differentiability corresponds to the existence of $\mathrm{gH}-$ differences as in (b) for $h \in] 0, \delta[, \delta>0$. When we have a switch, i.e. the gH-difference in (a) exists to the left while to the right the gH-difference according to (b) exists or viceversa, we obtain the GH-differentiability cases 
(iii) or (iv) in Definition 6. The fact that gH-differentiability is weaker than GH-differentiability will be also shown in a little while by an example.

Theorem 11. The gH-differentiability concept and the weakly generalized (Hukuhara) differentiability given in Definition 7 coincide.

PROOF. Indeed, let us suppose that $f$ is gH-differentiable (as in Definition 8). Since obviously, in the interval case for any sequence $h_{n} \searrow 0$ at least two of the Hukuhara differences $f\left(x_{0}+h_{n}\right) \ominus f\left(x_{0}\right), f\left(x_{0}\right) \ominus f\left(x_{0}+h_{n}\right), f\left(x_{0}\right) \ominus$ $f\left(x_{0}-h_{n}\right), f\left(x_{0}-h_{n}\right) \ominus f\left(x_{0}\right)$ exist, we have $A_{n_{0}}^{(1)} \cup A_{n_{0}}^{(2)} \cup A_{n_{0}}^{(3)} \cup A_{n_{0}}^{(4)}=\{n \in$ $\left.\mathbb{N} ; n \geq n_{0}\right\}$ for any $n_{0} \in \mathbb{N}$. The rest is obtained by observing that $\frac{E_{n}^{(j)}}{(-1)^{j+1} h_{n}}=$ $\frac{f\left(x_{0}+h_{n}\right) \ominus_{g} f\left(x_{0}\right)}{h_{n}}$, written with gH-difference this time. Reciprocally, if we assume $f$ to be weakly generalized (Hukuhara) differentiable then since at least two of the sets $A_{n_{0}}^{(1)}, A_{n_{0}}^{(2)}, A_{n_{0}}^{(3)}, A_{n_{0}}^{(4)}$ are infinite $\lim _{h \rightarrow 0} \frac{1}{h}\left[f\left(x_{0}+h\right) \ominus_{g} f\left(x_{0}\right)\right]=\lim _{h_{n} \searrow 0} \frac{E_{n}^{(j)}}{(-1)^{j+1} h_{n}}$ for at least two indices from $j \in\{1,2,3,4\}$, so $f$ is gH-differentiable. According to this remark, weakly generalized (Hukuhara) differentiability is equivalent to gH-differentiability.

The advantage of the Definition 8 is that we have a much simpler formulation and we do not have the four cases in Definition 7 explicitly for the weakly generalized (Hukuhara) differentiability concept; however implicitly we have all those cases so the differentiability concepts are exactly the same but formulated in a more compact way. So, from this point forward, the gHdifferentiability and the weakly generalized (Hukuhara) differentiability will be both denoted as gH-differentiability.

We also observe the intuitive interpretation of the gH-derivative as the rate of change with either increasing or decreasing uncertainty.

Example 12. ([14]) Let $A \neq\{\widehat{a}\}$ be a compact interval, $f(x)=x A, f(x+$ $h)=(x+h) A($ in general $\neq x A+h A)$ we always have $\frac{1}{h}\left[f\left(x_{0}+h\right) \ominus_{g} f\left(x_{0}\right)\right]=A$. Take $A=[-2,1]$ so

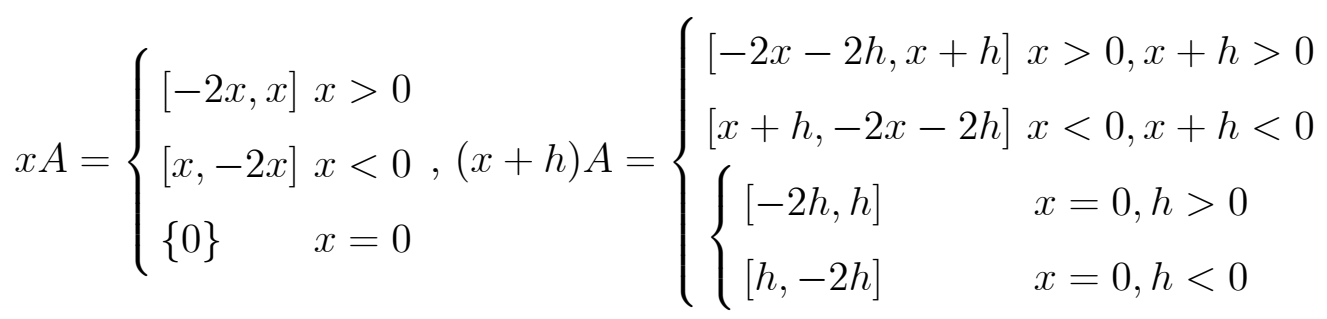


and

$$
\begin{gathered}
(x+h) A \ominus_{g} x A= \begin{cases}{[-2 h, h]} & x>0, x+h>0 \\
{[h,-2 h]} & x<0, x+h<0 \\
\begin{cases}{[-2 h, h]} & x=0, h>0 \\
{[h,-2 h]} & x=0, h<0\end{cases} \end{cases} \\
\text { So that } \\
\frac{1}{h}\left[(x+h) A \ominus_{g} x A\right]=[-2,1] \forall h \neq 0 .
\end{gathered}
$$

Example 13. (More general example): let $f(x)=p(x) A$ where $p$ is a crisp differentiable function and $A$ is an interval, then $f(x+h)=p(x+h) A$ and we obtain $f\left(x_{0}+h\right) \ominus_{g} f\left(x_{0}\right)=\left(p\left(x_{0}+h\right)-p\left(x_{0}\right)\right) A$; in fact, from

$$
\begin{aligned}
f\left(x_{0}+h\right) \ominus_{g} f\left(x_{0}\right) & =H\left(x_{0}, h\right) \\
& \begin{cases}\mathbb{1} \\
\text { either }(a) f\left(x_{0}+h\right)=f\left(x_{0}\right)+H\left(x_{0}, h\right)\end{cases}
\end{aligned}
$$

by examining separately the cases $\left(p\left(x_{0}\right)>0\right.$ and $h$ such that $\left.p\left(x_{0}+h\right)>0\right)$, $\left(p\left(x_{0}\right)<0\right.$ and $h$ such that $\left.p\left(x_{0}+h\right)<0\right),\left(p\left(x_{0}\right)=0\right.$ and $\left.p\left(x_{0}+h\right) \leq 0\right)$, $\left(p\left(x_{0}\right)=0\right.$ and $\left.p\left(x_{0}+h\right) \geq 0\right)$ we find either case (a) or case (b) of (1) and always $H\left(x_{0}, h\right)=\left(p\left(x_{0}+h\right)-p\left(x_{0}\right)\right) A$. It follows that $f^{\prime}\left(x_{0}\right)=p^{\prime}\left(x_{0}\right) A$.

The next result gives the expression of the gH-derivative in terms of the endpoints of the interval-valued function. This result is given in [7], Theorem 5 for the fuzzy-valued case considering only two cases of GH-differentiability in [6]. When we are dealing with interval-valued functions the reciprocal of the result in [7] is as well true.

Theorem 14. Let $f:[a, b] \longrightarrow \mathbb{I}$ be such that $f(x)=\left[f^{-}(x), f^{+}(x)\right]$. The function $f(x)$ is $g H$-differentiable if and only if $f^{-}(x)$ and $f^{+}(x)$ are differentiable real-valued functions and

$$
f^{\prime}(x)=\left[\min \left\{\left(f^{-}\right)^{\prime}(x),\left(f^{+}\right)^{\prime}(x)\right\}, \max \left\{\left(f^{-}\right)^{\prime}(x),\left(f^{+}\right)^{\prime}(x)\right\}\right] .
$$

PROOF. By [7], Theorem 5, if $f$ is gH-differentiable then $f^{-}(x)$ and $f^{+}(x)$ are differentiable and

$$
f^{\prime}(x)=\left[\min \left\{\left(f^{-}\right)^{\prime}(x),\left(f^{+}\right)^{\prime}(x)\right\}, \max \left\{\left(f^{-}\right)^{\prime}(x),\left(f^{+}\right)^{\prime}(x)\right\}\right] .
$$

Reciprocally, in the case of interval-valued functions, the gH-difference always exists. Analyzing all the possible cases of existence of the gH-differences to the 
left and right we obtain the reciprocal statement, i.e., if $f^{-}(x)$ and $f^{+}(x)$ are differentiable then $f$ is gH-differentiable and $f^{\prime}(x)=\left[\min \left\{\left(f^{-}\right)^{\prime}(x),\left(f^{+}\right)^{\prime}(x)\right\}\right.$, $\left.\max \left\{\left(f^{-}\right)^{\prime}(x),\left(f^{+}\right)^{\prime}(x)\right\}\right]$.

According to this theorem, for the definition of weakly generalized (Hukuhara) differentiability, we distinguish two cases, corresponding to (a) and (b) of (1).

Definition 15. Let $f:[a, b] \longrightarrow \mathbb{I}$ be gH-differentiable at $\left.x_{0} \in\right] a, b[$. We say that $f$ is (i)-gH-differentiable at $x_{0}$ if

$$
\text { (i.) } \quad f^{\prime}\left(x_{0}\right)=\left[\left(f^{-}\right)^{\prime}\left(x_{0}\right),\left(f^{+}\right)^{\prime}\left(x_{0}\right)\right]
$$

and that $f$ is (ii)-gH-differentiable at $x_{0}$ if

$$
\text { (ii.) } \quad f^{\prime}\left(x_{0}\right)=\left[\left(f^{+}\right)^{\prime}\left(x_{0}\right),\left(f^{-}\right)^{\prime}\left(x_{0}\right)\right] \text {. }
$$

Remark 16. According to the previous results we can see that the Definitions 7, 8 and 15 are equivalent, so we can chose the most convenient formulation depending on the application at hand.

Remark 17. We observe that if $f$ is GH-differentiable then cases (i) and (ii) of gH-differentiability are in fact cases (i) and (ii) of the GH-differentiability provided that the same case remains in effect on an interval of non-zero length. According to a result in [6] related to GH-differentiability the cases (iii) and (iv) of Definition 6 can happen only on a discrete set of points. Also, let us remark here that if a function is GH-differentiable it is obviously $\mathrm{gH}$ differentiable too, and the two derivatives coincide. So, combining these results we can see that if a function is gH-differentiable and if there exists a partition $a=c_{1} \leq c_{2} \leq \ldots \leq c_{n}=b$ such that exactly one of the cases (i) or (ii) is kept over any interval $\left[x_{i}, x_{i+1}\right]$ (without the possibility of a switch in $] x_{i}, x_{i+1}[$ ), then $f$ is GH-differentiable.

Remark 18. In [7] the cases (i.) and (ii.) are said to be the lateral derivatives (in fact they are cases (i) and (ii) of the GH-differentiability).

Since in definitions 8 and 15 the requirement that we keep the same case of existence (a) or (b) for the gH-difference over an interval is released, these concepts are slightly more general than the strongly generalized (Hukuhara) differentiability. This is also shown by the following example.

Example 19. Let us consider the function $f: \mathbb{R} \rightarrow \mathbb{I}$,

$$
f(x)=\left\{\begin{array}{l}
{[-1,1] \cdot\left(1-x^{2} \sin \frac{1}{x}\right) \text { if } x \neq 0} \\
{[-1,1], \text { otherwise }}
\end{array}\right.
$$


It is easy to check by Theorem 14 that $f$ is gH-differentiable at 0 and $f^{\prime}(0)=0$, ( 0 in the right denotes the singleton $\{0\})$. Also, we observe that $f$ is not GHdifferentiable since there does not exist $\delta>0$ such that $f(h) \ominus f(0)$ or $f(-h) \ominus f(0)$ exists for all $h \in(0, \delta)$.

The following properties are obtained from Theorem 14.

Proposition 20. Let $f, g:[a, b] \longrightarrow \mathbb{I}$ be such that $f(x)=\left[f^{-}(x), f^{+}(x)\right]$ and $g(x)=\left[g^{-}(x), g^{+}(x)\right]$ are $g H$-differentiable. Then

(i) $f+g$ is $g H$-differentiable and

$$
\begin{aligned}
&(f+g)^{\prime}(x)=\left[\min \left\{\left(f^{-}+g^{-}\right)^{\prime}(x),\left(f^{+}+g^{+}\right)^{\prime}(x)\right\}\right. \\
&\left.\max \left\{\left(f^{-}+g^{-}\right)^{\prime}(x),\left(f^{+}+g^{+}\right)^{\prime}(x)\right\}\right] .
\end{aligned}
$$

(ii) if $f \cdot g$ is the product in the usual interval arithmetic then $f \cdot g$ is $g H$ differentiable and

$$
\begin{gathered}
(f \cdot g)^{\prime}(x)=\left[\min \left\{\left(f^{-} \cdot g^{-}\right)^{\prime}(x),\left(f^{-} \cdot g^{+}\right)^{\prime}(x),\left(f^{+} \cdot g^{-}\right)^{\prime}(x),\left(f^{+} \cdot g^{+}\right)^{\prime}(x)\right\}\right. \\
\left.\max \left\{\left(f^{-} \cdot g^{-}\right)^{\prime}(x),\left(f^{-} \cdot g^{+}\right)^{\prime}(x),\left(f^{+} \cdot g^{-}\right)^{\prime}(x),\left(f^{+} \cdot g^{+}\right)^{\prime}(x)\right\}\right] .
\end{gathered}
$$

Remark 21. If $k$ is a constant then $(k f)^{\prime}=k f^{\prime}$ but, in general, we have the following inclusions:

$$
(f+g)^{\prime} \supseteq f^{\prime}+g^{\prime} \text { and }(f g)^{\prime} \supseteq f^{\prime} g+f g^{\prime} .
$$

It is an interesting problem to see how the switch between the two cases can occur.

Definition 22. We say that a point $\left.x_{0} \in\right] a, b[$ is an l-critical point if with respect to (2), at the points $x_{0}$ the gH-differentiability case switches from (i) to (ii) in (3)-(4) or from (ii) to (i) in (4)-(3).

We call these points as l-critical points of $f(x)$ since they are critical points of the function $\operatorname{len}(f(x))=f^{+}(x)-f^{-}(x)$ which gives the length of the interval $f(x)$.

Definition 23. Let $\widehat{x} \in] a, b[$ be an l-critical point of a gH-differentiable function $f:[a, b] \longrightarrow \mathbb{I}$. We say that $\widehat{x}$ is a type-I switching point for $f(x)$ if $\exists \delta>0$ such that for $|x-\widehat{x}|<\delta$

$$
\begin{aligned}
& f^{\prime}(x)=\left[\left(f^{-}\right)^{\prime}(x),\left(f^{+}\right)^{\prime}(x)\right] \text { if } x \leq \widehat{x}, \\
& f^{\prime}(x)=\left[\left(f^{+}\right)^{\prime}(x),\left(f^{-}\right)^{\prime}(x)\right] \text { if } x \geq \widehat{x}
\end{aligned}
$$


and we say that $\widehat{x}$ is a type-II switching point for $f(x)$ if $\exists \delta>0$ such that for $|x-\widehat{x}|<\delta$

$$
\begin{aligned}
& f^{\prime}(x)=\left[\left(f^{+}\right)^{\prime}(x),\left(f^{-}\right)^{\prime}(x)\right] \text { if } x \leq \widehat{x}, \\
& f^{\prime}(x)=\left[\left(f^{-}\right)^{\prime}(x),\left(f^{+}\right)^{\prime}(x)\right] \text { if } x \geq \widehat{x} .
\end{aligned}
$$

Clearly, not all $l$-critical points are also switching points, as illustrated in the following example.

Example 24. Consider the interval valued functions $f_{1}(x)=\left[\frac{1}{2} x^{2}, 1+\frac{1}{2} x^{2}+\right.$ $\left.2 \sin ^{2}(x)\right], f_{2}(x)=\left[\frac{1}{2} x^{2}, 1+x+\frac{1}{2} x^{2}+2 \sin ^{2}(x)\right] x \in[0,2 \pi]$. Function $f_{1}$ has three l-critical points in ]0,2 $\pi$ [ and they are switching points $\widehat{x}_{1}=\frac{1}{2} \pi$ of typeII, $\widehat{x}_{2}=\pi$ of type-I and $\widehat{x}_{3}=\frac{3}{2} \pi$ of type-II; $\widehat{x}_{1}$ and $\widehat{x}_{3}$ correspond to the maxima and $\widehat{x}_{2}$ to the minimum points of $\operatorname{len}\left(f_{1}(x)\right)$.

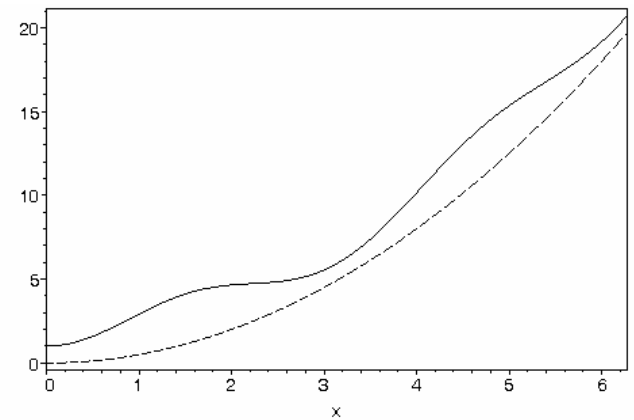

Figure 1a. Interval valued function $f_{1}(x)$.

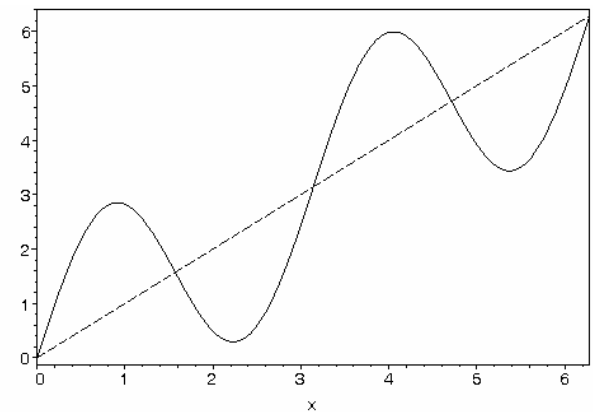

Figure 1b. Interval valued derivatives $\left(f_{1}^{-}\right)^{\prime}(x),\left(f_{1}^{+}\right)^{\prime}(x)$.

Function $f_{2}$ has two l-critical points $\widehat{x}_{1}=\frac{3}{4} \pi, \widehat{x}_{2}=\frac{7}{4} \pi$ in $] 0,2 \pi[$ but they are not switching points; $\widehat{x}_{1}$ and $\widehat{x}_{2}$ correspond to horizontal inflection points of 
function $\operatorname{len}\left(f_{2}(x)\right)$.

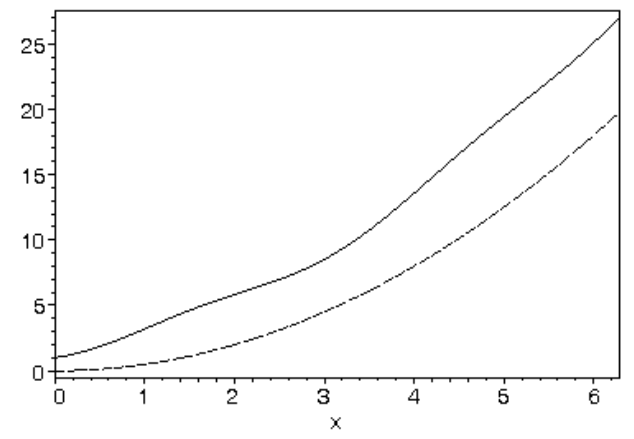

Figure 2a. Interval valued function $f_{2}(x)$.

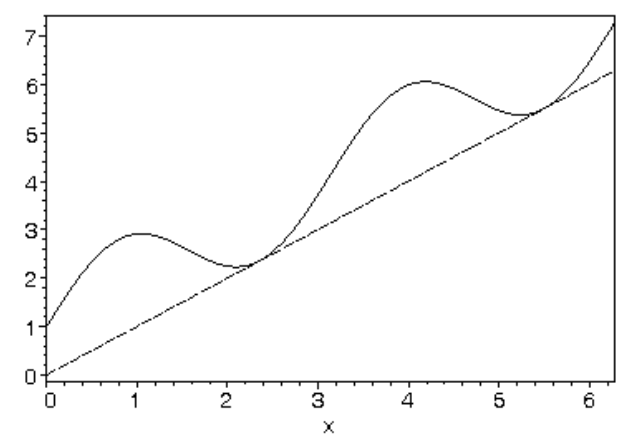

Figure 2b. Interval valued derivatives $\left(f_{2}^{-}\right)^{\prime}(x),\left(f_{2}^{+}\right)^{\prime}(x)$.

Finally we can collect all the differentiability concepts together in the following theorem.

Theorem 25. Let $f:] a, b\left[\rightarrow \mathbb{I}\right.$ is a function $f(x)=\left[f^{-}(x), f^{+}(x)\right]$. The following affirmations are equivalent:

(1) $f$ is GH-differentiable

(2) $f$ is gH-differentiable and the set of l-critical points is finite.

PROOF. According to a result in [6], if a function is GH-differentiable, the switches form a discrete set of points. Moreover, at these points we have $\left(f^{-}\right)^{\prime}(\widehat{x})=\left(f^{+}\right)^{\prime}(\widehat{x})$, i.e. in this case $f^{\prime}(\widehat{x})$ is necessarily a singleton and these points are exactly the $l$-critical points. Reciprocally, if $f$ is gH-differentiable and the set of $l$-critical points is finite $\left\{c_{2}, \ldots, c_{n-1}\right\}$ then we can find a partition $a=c_{1} \leq c_{2} \leq \ldots \leq c_{n}=b$ such that exactly one of the cases (i) or (ii) is kept over any interval $\left[x_{i}, x_{i+1}\right]$, thus $f$ will be GH-differentiable. 
In the following sections basically we will allow only a finite number of $l$-critical points. So from this point forward in the present paper GH-differentiability and gH-differentiability concepts are identical (with the restriction that we may have only a finite number of $l$-critical points). In fact, the requirement that the number of $l$-critical points is finite is not very restrictive, we could see an example where this condition was not fulfilled, but the function there was not one of those which appear frequently in applications. So, from now on we will use the GH-differentiability concept or equivalently the gH-differentiability with a finite number of $l$-critical points.

\section{Integration of interval valued functions}

In the present paper the integral of an interval valued function $f:[a, b] \longrightarrow \mathbb{I}$ with $f(x)=\left[f^{-}(x), f^{+}(x)\right]$ is defined as usually:

$$
\int_{a}^{b} f(x) d x=\left[\int_{a}^{b} f^{-}(x) d x, \int_{a}^{b} f^{+}(x) d x\right] .
$$

The Newton-Leibnitz formula can be extended to the interval case with some caution. The changes in the cases of existence of the gH-differences imply that we do not have a straightforward extension of the classical formula for the interval case.

Theorem 26. Let $f:[a, b] \rightarrow \mathbb{I}$ be continuous. Then

(i) the function $F(x)=\int_{a}^{x} f(t) d t$ is differentiable and $F^{\prime}(x)=f(x)$,

(ii) the function $G(x)=\int_{x}^{b} f(t) d t$ is differentiable and $G^{\prime}(x)=-f(x)$.

PROOF. We have $F(x)=\int_{a}^{x} f(t) d t=\left[F^{-}(x), F^{+}(x)\right]$ and $G(x)=\int_{x}^{b} f(t) d t=$ $\left[G^{-}(x), G^{+}(x)\right]$. Then

$$
\begin{aligned}
& F^{\prime-}\left(x_{0}\right)=\min \left\{f^{-}\left(x_{0}\right), f^{+}\left(x_{0}\right)\right\}=f^{-}\left(x_{0}\right) \\
& F^{\prime+}\left(x_{0}\right)=\max \left\{f^{-}\left(x_{0}\right), f^{+}\left(x_{0}\right)\right\}=f^{+}\left(x_{0}\right)
\end{aligned}
$$

and

$$
\begin{aligned}
& G^{\prime-}\left(x_{0}\right)=\min \left\{-f^{-}\left(x_{0}\right),-f^{+}\left(x_{0}\right)\right\}=-f^{+}\left(x_{0}\right) \\
& G^{\prime+}\left(x_{0}\right)=\max \left\{-f^{-}\left(x_{0}\right),-f^{+}\left(x_{0}\right)\right\}=-f^{-}\left(x_{0}\right) .
\end{aligned}
$$


Theorem 27. Let us suppose that function $f$ changes differentiability case only a finite number of times, at given points $a=c_{0}<c_{1}<c_{2}<\ldots<c_{n}<$ $c_{n+1}=b$ and exactly at these points. Then we have

$$
f(b) \ominus_{g} f(a)=\sum_{i=1}^{n}\left[\int_{c_{i-1}}^{c_{i}} f^{\prime}(x) d x \ominus_{g}(-1) \int_{c_{i}}^{c_{i}+1} f^{\prime}(x) d x\right]
$$

Also,

$$
\int_{a}^{b} f^{\prime}(x) d x=\sum_{i=1}^{n+1}\left[f\left(c_{i}\right) \Theta_{g} f\left(c_{i-1}\right)\right]
$$

and $\int_{a}^{b} f^{\prime}(x) d x=f(b) \Theta_{g} f(a)$ if and only if $f\left(c_{i}\right)$ is crisp for $i=1,2, \ldots, n$.

PROOF. For simplicity we consider only one switch point, the case of a finite number of switch-points follows similarly. Let us suppose that $f$ is (i)differentiable on $[a, c]$ and (ii)-differentiable on $[c, b]$. Then we have by direct calculation

$$
\begin{aligned}
\int_{a}^{c} f^{\prime}(x) d x & \Theta_{g}(-1) \int_{c}^{b} f^{\prime}(x) d x=\left(f(c) \Theta_{g} f(a)\right) \Theta_{g}(-1)\left(f(b) \Theta_{g} f(c)\right) \\
& =(f(c) \ominus f(a)) \Theta_{g}(f(c) \ominus f(b)) \\
& =\left[f^{-}(c)-f^{-}(a), f^{+}(c)-f^{+}(a)\right] \ominus_{g}\left[f^{-}(c)-f^{-}(b), f^{+}(c)-f^{+}(b)\right] \\
& =f(b) \Theta_{g} f(a) .
\end{aligned}
$$

Also, it is easy to check that

$$
\begin{aligned}
\int_{a}^{b} f^{\prime}(x) d x & =\int_{a}^{c} f^{\prime}(x) d x+\int_{c}^{b} f^{\prime}(x) d x= \\
& =\left(f(c) \ominus_{g} f(a)\right)+\left(f(b) \ominus_{g} f(c)\right) .
\end{aligned}
$$

and $\left(f(c) \Theta_{g} f(a)\right)+\left(f(b) \Theta_{g} f(c)\right)=f(b) \Theta_{g} f(a)$ if and only if $f(c)$ is a singleton (note that for a switching point $c$ we cannot have $f(a)=f(c)=f(b)$ i.e. $f^{-}(a)=f^{-}(c)=f^{-}(b)$ and $\left.f^{+}(a)=f^{+}(c)=f(b)\right)$.

Corollary 28. If $f$ does not change differentiability case on the interval $[a, b]$ then we have

$$
\int_{a}^{b} f^{\prime}(x) d x=f(b) \ominus_{g} f(a) .
$$

Remark 29. Generally, the statement of the corollary does not hold. Indeed, if we use the usual Hukuhara difference we have

$$
\begin{aligned}
& \left(f(c) \Theta_{g} f(a)\right)+\left(f(b) \Theta_{g} f(c)\right) \\
& =f(c) \ominus f(a)+(-1)(f(c) \Theta f(b)) \\
& =\left[f^{-}(c)-f^{-}(a)-f^{+}(c)+f^{+}(b), f^{+}(c)-f^{+}(a)-f^{-}(c)+f^{-}(b)\right] \\
& \neq f(b) \Theta_{g} f(a) .
\end{aligned}
$$


However we know that $f^{\prime}(c) \in \mathbb{R}$ i.e., $\left(f^{-}\right)^{\prime}(c)=\left(f^{+}\right)^{\prime}(c)$ and we cannot conclude anything for $f^{-}(c)$ and $f^{+}(c)$.

\section{Interval differential equations}

In this section we consider an interval valued differential equation

$$
\begin{gathered}
\qquad \begin{array}{c}
y^{\prime} \\
=f(x, y), y\left(x_{0}\right)=y_{0} \\
\text { where } \\
f:[a, b] \times \mathbb{I} \longrightarrow \mathbb{I} \\
\text { with } f(x, y)=\left[f^{-}(x, y), f^{+}(x, y)\right] \text { for } y \in \mathbb{I} \\
y=\left[y^{-}, y^{+}\right], y_{0}=\left[y_{0}^{-}, y_{0}^{+}\right] .
\end{array}
\end{gathered}
$$

The following Lemma is a consequence of the Newton Leibnitz-type formulas discussed in the previous section.

Lemma 30. The interval differential equation (7) is locally equivalent to the integral equation

$$
y(x) \ominus_{g} y_{0}=\int_{x_{0}}^{x} f(t, y(t)) d t
$$

The two cases of existence of the gH-difference imply that the integral equation in the Lemma is actually a unified formulation for one of the integral equations

$$
y(x) \ominus y_{0}=\int_{x_{0}}^{x} f(t, y(t)) d t
$$

and

$$
y_{0} \ominus y(x)=-\int_{x_{0}}^{x} f(t, y(t)) d t
$$

with $\ominus$ being the classical Hukuhara difference.

By using the integral equation formulation we obtain an existence result analogous to the result in [6]. In the interval case the result is simplified significantly.

Theorem 31. Let $R_{0}=\left[x_{0}, x_{0}+p\right] \times \mathbb{I}, y_{0} \in \mathbb{I}$ nontrivial and $f: R_{0} \rightarrow \mathbb{I}$ be continuous. If $f$ satisfies the Lipschitz condition $D(f(x, y), f(x, z)) \leq L$. $D(y, z), \forall(x, y),(x, z) \in R_{0}$ then the interval problem

$$
\left\{\begin{array}{c}
y^{\prime}=f(x, y) \\
y\left(x_{0}\right)=y_{0}
\end{array}\right.
$$


has exactly two solutions $y, \bar{y}:\left[x_{0}, x_{0}+r\right] \rightarrow \bar{B}\left(y_{0}, q\right)$ satisfying

$$
\begin{gathered}
y_{0}(x)=y_{0} \\
y_{n+1}(x) \ominus_{g} y_{0}=\int_{x_{0}}^{x} f\left(t, y_{n}(t)\right) d t
\end{gathered}
$$

More precisely, the successive iterations

$$
\begin{aligned}
y_{n+1}(x)= & y_{0}+\int_{x_{0}}^{x} f\left(t, y_{n}(t)\right) d t \quad \text { i.e. } \\
(\text { a }) & \left\{\begin{array}{l}
y_{n+1}^{-}(x)=y_{0}^{-}+\int_{x_{0}}^{x} f^{-}\left(t, y_{n}(t)\right) d t \\
y_{n+1}^{+}(x)=y_{0}^{+}+\int_{x_{0}}^{x} f^{+}\left(t, y_{n}(t)\right) d t
\end{array}\right.
\end{aligned}
$$

and

$$
\begin{gathered}
y_{0}=\bar{y}_{n+1}(x)-\int_{x_{0}}^{x} f\left(t, \bar{y}_{n}(t)\right) d t \quad \text { i.e. } \\
\text { (b) }\left\{\begin{array}{l}
\bar{y}_{n+1}^{-}(x)=y_{0}^{-}+\int_{x_{0}}^{x} f^{+}\left(t, \bar{y}_{n}(t)\right) d t \\
\bar{y}_{n+1}^{+}(x)=y_{0}^{+}+\int_{x_{0}}^{x} f^{-}\left(t, \bar{y}_{n}(t)\right) d t
\end{array}\right.
\end{gathered}
$$

converge to these two solutions $y$ and $\bar{y}$ respectively.

PROOF. The proof is similar to [6] Theorems 22 and 25 (see also [42]). The boundedness condition in these above cited theorems can be released since the space of intervals in contrast to the space of fuzzy numbers is locally compact with respect to the Hausdorff distance. So, the boundedness conditions in fuzzy setting now follows by the continuity of $f$ and the fact that a closed ball is a compact subspace of $R_{0}$. The condition (4) of [6], Theorem 22 , is fulfilled since $y_{0}$ is a nontrivial interval.

In what follows we would like to obtain a characterization theorem for interval differential equations by ODEs, similar to [5]. So, we consider the interval differential equation (7) with gH-differentiability.

As

$$
y(x) \ominus_{g} y_{0}=\left[\min \left\{y^{-}(x)-y_{0}^{-}, y^{+}(x)-y_{0}^{+}\right\}, \max \left\{y^{-}(x)-y_{0}^{-}, y^{+}(x)-y_{0}^{+}\right\}\right]
$$

we have the integral equations

$$
\left\{\begin{array}{l}
\min \left\{y^{-}(x)-y_{0}^{-}, y^{+}(x)-y_{0}^{+}\right\}=\int_{x_{0}}^{x} f^{-}(t, y(t)) d t \\
\max \left\{y^{-}(x)-y_{0}^{-}, y^{+}(x)-y_{0}^{+}\right\}=\int_{x_{0}}^{x} f^{+}(t, y(t)) d t .
\end{array}\right.
$$


Considering the differential form, we obtain two differential equations

$$
\left\{\begin{array}{l}
\min \left\{y^{\prime-}(x), y^{\prime+}(x)\right\}=f^{-}(x, y(x)) \\
\max \left\{y^{\prime-}(x), y^{\prime+}(x)\right\}=f^{+}(x, y(x)) \\
\text { s.t. } \\
y^{-}\left(x_{0}\right)=y_{0}^{-} \\
y^{+}\left(x_{0}\right)=y_{0}^{+} .
\end{array}\right.
$$

Note that, in general, $f^{-}(x, y)$ and $f^{+}(x, y)$ are functions of the interval $y=$ $\left[y^{-}, y^{+}\right]$. Then we set

$$
\left\{\begin{array}{l}
\varphi^{-}\left(x, y^{-}, y^{+}\right)=f^{-}(x, y) \\
\varphi^{+}\left(x, y^{-}, y^{+}\right)=f^{+}(x, y)
\end{array}\right.
$$

with $\varphi^{-} \leq \varphi^{+}$defined on a subset of $\mathbb{R}^{3}$.

Finally, we obtain two situations:

ODE(a): $y^{\prime-}(x) \leq y^{\prime+}(x)$; the differential equations are

$$
\left\{\begin{array}{l}
y^{\prime-}(x)=\varphi^{-}\left(x, y^{-}(x), y^{+}(x)\right) \\
y^{\prime+}(x)=\varphi^{+}\left(x, y^{-}(x), y^{+}(x)\right) \\
\text { s.t. } \\
y^{-}\left(x_{0}\right)=y_{0}^{-} \\
y^{+}\left(x_{0}\right)=y_{0}^{+}
\end{array}\right.
$$

and if $\varphi^{-}$depends only on $y^{-}$and $\varphi^{+}$depends only on $y^{+}$, the two equations are independent.

$\mathrm{ODE}(\mathrm{b}): y^{-}(x) \geq y^{\prime+}(x)$; the differential equations are

$$
\left\{\begin{array}{l}
y^{\prime-}(x)=\varphi^{+}\left(x, y^{-}(x), y^{+}(x)\right) \\
y^{\prime+}(x)=\varphi^{-}\left(x, y^{-}(x), y^{+}(x)\right) \\
\text { s.t. } \\
y^{-}\left(x_{0}\right)=y_{0}^{-} \\
y^{+}\left(x_{0}\right)=y_{0}^{+}
\end{array}\right.
$$

and if $\varphi^{+}$depends only on $y^{-}$and $\varphi^{-}$depends only on $y^{+}$, the two equations are independent. 
Now we present a characterization result:

Theorem 32. Let $R_{0}=\left[x_{0}, x_{0}+p\right] \times \mathbb{I}, y_{0} \in \mathbb{I}$ nontrivial and $f: R_{0} \rightarrow \mathbb{I}$ be continuous. If $f$ satisfies the Lipschitz condition $D(f(x, y), f(x, z)) \leq L$. $D(y, z), \forall(x, y),(x, z) \in R_{0}$ then the interval problem

$$
\left\{\begin{array}{c}
y^{\prime}=f(x, y) \\
y\left(x_{0}\right)=y_{0}
\end{array}\right.
$$

is equivalent to union of the ODEs (9) and (10) on some interval $\left[x_{0}, x_{0}+q\right]$.

PROOF. The conditions of the theorem ensure the existence and uniqueness of two solutions for the interval IVP, one of the solutions being differentiable in the case (i.) the other in case (ii.) of gH-differentiability. By Theorem 14 the solutions obtained via $\mathrm{ODE}(\mathrm{a})$ and $\mathrm{ODE}(\mathrm{b})$, respectively, correspond (locally) to a solution of (a) and (b) of Theorem 31. Reciprocally, the continuity and the Lipschitz property of $f$ ensures a Lipschitz continuity for the functions $\varphi^{-}, \varphi^{+}$. So, each of the equations (9) and (10) have a unique solution respectively $y_{1}$ and $y_{2}$. Moreover, since the interval $y_{0}$ is nontrivial there exists a $q>0$ such that for $x \in\left[x_{0}, x_{0}+q\right]$ the solution of (10) satisfies $y_{2}^{\prime-}(x) \geq y_{2}^{\prime+}(x)$. Also, the conditions in the theorem are enough for the existence and uniqueness of two solutions of the problem (11) $\tilde{y}_{1}, \tilde{y}_{2}$. If $\tilde{y}_{1}$ is the usual Hukuhara solution then $\tilde{y}_{1}$ is Hukuhara differentiable and then it is a solution of (9); if $\tilde{y}_{2}$ is the solution in the case (b), then $\tilde{y}_{2}$ is gH-differentiable and it is a solution of (10).

All the preceding results hold for general interval-valued functions so possibly they depend on interval parameters, i.e., interval extensions of real-valued functions of the form $f\left(x, y, p_{1}, \ldots, p_{n}\right)$ with $p_{i} \in P_{i} \in \mathbb{I}$.

Let us analyze the case when $f(x, y)$ is the interval extension of a real valued function $f(x, p)$, i.e.,

$$
f(x, y)=\left[\inf _{p \in y} f(x, p), \sup _{p \in y} f(x, p)\right]
$$

Let us suppose further that $f$ is monotonic with respect to $p$. 
1) if $f(x, p)$ is increasing with respect to $p$,

$$
\begin{array}{ll}
\text { case }(a): y^{\prime-}(x) \leq y^{\prime+}(x) & \text { case }(b): y^{\prime-}(x) \geq y^{\prime+}(x) \\
\left\{\begin{array} { l } 
{ y ^ { \prime - } ( x ) = f ( x , y ^ { - } ( x ) ) } \\
{ y ^ { \prime + } ( x ) = f ( x , y ^ { + } ( x ) ) , } \\
{ \text { s.t. } y ^ { - } ( x _ { 0 } ) = y _ { 0 } ^ { - } , } \\
{ y ^ { + } ( x _ { 0 } ) = y _ { 0 } ^ { + } }
\end{array} \quad \left\{\begin{array}{l}
y^{\prime+}(x)=f\left(x, y^{-}(x)\right) \\
y^{\prime-}(x)=f\left(x, y^{+}(x)\right), \\
\text { s.t. } y^{-}\left(x_{0}\right)=y_{0}^{-}, \\
y^{+}\left(x_{0}\right)=y_{0}^{+}
\end{array}\right.\right.
\end{array}
$$

2 ) if $f(x, p)$ is decreasing with respect to $p$

$$
\begin{array}{ll}
\text { case }(a): y^{\prime-}(x) \leq y^{\prime+}(x) & \text { case }(b): y^{\prime-}(x) \geq y^{\prime+}(x) \\
\left\{\begin{array} { l } 
{ y ^ { \prime - } ( x ) = f ( x , y ^ { + } ( x ) ) } \\
{ y ^ { \prime + } ( x ) = f ( x , y ^ { - } ( x ) ) , } \\
{ \text { s.t. } y ^ { - } ( x _ { 0 } ) = y _ { 0 } ^ { - } , } \\
{ y ^ { + } ( x _ { 0 } ) = y _ { 0 } ^ { + } }
\end{array} \quad \left\{\begin{array}{l}
y^{\prime+}(x)=f\left(x, y^{+}(x)\right) \\
y^{\prime-}(x)=f\left(x, y^{-}(x)\right), \\
\text { s.t. } y^{-}\left(x_{0}\right)=y_{0}^{-}, \\
y^{+}\left(x_{0}\right)=y_{0}^{+}
\end{array}\right.\right.
\end{array}
$$

Also, in this case, a very interesting result from [7] connects these cases to differential inclusions. In the followings we formulate a particularization of the result of Chalco-Cano and Roman-Flores. Namely we have:

Theorem 33. [7] Let $f(x, p)$ be a monotonic function with respect to $p \in \mathbb{R}$ and let $y_{0} \in \mathbb{I}$.

a) If $f(x, y)$ is the interval extension of $f$ and $f$ is an increasing function, then the solution in case (a) of (12) and the attainable set of the differential inclusion $y^{\prime}=f(x, y), y\left(x_{0}\right) \in y_{0}$ coincide locally.

b) If $f(x, y)$ is the interval extension of $f$ and $f$ is a decreasing function, then the solution in case (b) of (13) and the attainable set of the differential inclusion $y^{\prime}=f(x, y), y\left(x_{0}\right) \in y_{0}$ coincide locally whenever $y_{0} \in \mathbb{I}$ is nontrivial.

We can have more general situations not covered by the above theorem. Indeed, let $f(x, y)=\exp \left(-y^{2}\right)$ as interval extension of $\exp \left(-p^{2}\right)$ to $y=\left[y^{-}, y^{+}\right]$. Then 
since the function $f$ is not monotonic we will have a more general situation:

$$
\begin{gathered}
\varphi^{-}\left(x, y^{-}, y^{+}\right)=f^{-}(x, y)=\left\{\begin{array}{lr}
\exp \left(-\left(y^{-}\right)^{2}\right) & \text { if } y^{+} \leq 0 \\
\exp \left(-\left(y^{+}\right)^{2}\right) & \text { if } y^{-} \geq 0 \\
\min \left\{\exp \left(-\left(y^{-}\right)^{2}\right), \exp \left(-\left(y^{+}\right)^{2}\right)\right\} & \text { otherwise }
\end{array}\right. \\
\varphi^{+}\left(x, y^{-}, y^{+}\right)=f^{+}(x, y)= \begin{cases}\exp \left(-\left(y^{+}\right)^{2}\right) & \text { if } y^{+} \leq 0 \\
\exp \left(-\left(y^{-}\right)^{2}\right) & \text { if } y^{-} \geq 0 \\
1 & \text { otherwise. }\end{cases}
\end{gathered}
$$

The connection between the gH-differentiability approach to IDEs and differential inclusions is another subject of further study. This connection is helpful since it leads to simple numerical algorithms for differential inclusions. We will use the previous Theorem for solving practically some particular types of differential inclusions, but a subject of future study is surely, how this extends to more general situations with differential inclusions.

\section{Interval differential equations by gH-derivative and differential algebraic equations}

In this section we discuss a connection between interval differential equations (IDE) and differential algebraic equations (DAE).

Let us denote $f(x, y)=\left[f^{-}(x, y), f^{+}(x, y)\right]$ the interval valued function at the right hand side of the IDE

$$
\begin{aligned}
& y^{\prime}(x)=f(x, y(x)) \text { for } x \in\left[x_{0}, X\right] \\
& \text { with initial condition } \\
& y\left(x_{0}\right)=y_{0}=\left[u_{0}, v_{0}\right] \text { given at } x=x_{0} .
\end{aligned}
$$

For each $x \in\left[x_{0}, X\right]$, denote by $y(x)=[u(x), v(x)]$ the interval valued solution of (14), so that functions $\varphi^{-}$and $\varphi^{+}$in (8) are now defined by

$$
\left\{\begin{array}{l}
\varphi^{-}(x, u(x), v(x))=f^{-}(x,[u(x), v(x)]) \\
\varphi^{+}(x, u(x), v(x))=f^{+}(x,[u(x), v(x)])
\end{array}\right.
$$

Theorem 34. Let $R_{0}=\left[x_{0}, x_{0}+p\right] \times \mathbb{I}, y_{0} \in \mathbb{I}$ nontrivial and $f: R_{0} \rightarrow \mathbb{I}$ be continuous. If $f$ satisfies the Lipschitz condition $D(f(x, y), f(x, z)) \leq L$. 
$D(y, z), \forall(x, y),(x, z) \in R_{0}$ then the interval problem

$$
\left\{\begin{array}{c}
y^{\prime}=f(x, y) \\
y\left(x_{0}\right)=y_{0}
\end{array}\right.
$$

is equivalent to the differential algebraic system (with $\varphi^{-}$and $\varphi^{+}$defined in )

$$
\left\{\begin{aligned}
u^{\prime}(x)+v^{\prime}(x) & =\varphi^{+}(x, u(x), v(x))+\varphi^{-}(x, u(x), v(x)) \\
\left|u^{\prime}(x)-v^{\prime}(x)\right| & =\varphi^{+}(x, u(x), v(x))-\varphi^{-}(x, u(x), v(x)) .
\end{aligned}\right.
$$

PROOF. Let us suppose that $y(x)$ it is gH-differentiable (by using the $\mathrm{gH}$ difference); then it follows that

$$
\begin{gathered}
y^{\prime}(x)=\left[\left(y^{\prime}(x)\right)^{-},\left(y^{\prime}(x)\right)^{+}\right] \\
\text {with } \\
\left(y^{\prime}(x)\right)^{-}=\min \left\{u^{\prime}(x), v^{\prime}(x)\right\} \\
\left(y^{\prime}(x)\right)^{+}=\max \left\{u^{\prime}(x), v^{\prime}(x)\right\} .
\end{gathered}
$$

We can write (18), equivalently, as $(\min +\max =$ sum, $\max -\min =$ absolute difference)

$$
\left\{\begin{array}{c}
\left(y^{\prime}(x)\right)^{-}+\left(y^{\prime}(x)\right)^{+}=u^{\prime}(x)+v^{\prime}(x) \\
\left(y^{\prime}(x)\right)^{+}-\left(y^{\prime}(x)\right)^{-}=\left|u^{\prime}(x)-v^{\prime}(x)\right|
\end{array}\right.
$$

and (16), taking (15), as

$$
\begin{aligned}
& \left(y^{\prime}(x)\right)^{-}=\varphi^{-}(x, u(x), v(x)) \\
& \left(y^{\prime}(x)\right)^{+}=\varphi^{+}(x, u(x), v(x))
\end{aligned}
$$

so that it becomes (substitute with sum and difference so we have invertibility for $\left(y^{\prime}(x)\right)^{-}$and $\left.\left(y^{\prime}(x)\right)^{+}\right)$

$$
\begin{aligned}
u^{\prime}(x)+v^{\prime}(x) & =\varphi^{+}(x, u(x), v(x))+\varphi^{-}(x, u(x), v(x)) \\
\left|u^{\prime}(x)-v^{\prime}(x)\right| & =\varphi^{+}(x, u(x), v(x))-\varphi^{-}(x, u(x), v(x)) .
\end{aligned}
$$

Equation (17) has a special form and the presence of the absolute value produces the following two cases for the second equation:

$$
\left\{\begin{array}{l}
u^{\prime}(x)-v^{\prime}(x)=\varphi^{+}(x, u(x), v(x))-\varphi^{-}(x, u(x), v(x)) \text { if } u^{\prime}(x) \geq v^{\prime}(x) \\
v^{\prime}(x)-u^{\prime}(x)=\varphi^{+}(x, u(x), v(x))-\varphi^{-}(x, u(x), v(x)) \text { if } u^{\prime}(x) \leq v^{\prime}(x)
\end{array}\right.
$$

taking into account the first of (17) and substituting $v^{\prime}(x)$, given by

$$
v^{\prime}(x)=\varphi^{+}(x, u(x), v(x))+\varphi^{-}(x, u(x), v(x))-u^{\prime}(x),
$$


we obtain the two ODEs:

$$
\begin{aligned}
& \text { if } u^{\prime}(x) \leq v^{\prime}(x):\left\{\begin{array}{l}
u^{\prime}(x)=\varphi^{-}(x, u(x), v(x)) \\
v^{\prime}(x)=\varphi^{+}(x, u(x), v(x))
\end{array}\right. \\
& \text { if } u^{\prime}(x) \geq v^{\prime}(x):\left\{\begin{array}{l}
u^{\prime}(x)=\varphi^{+}(x, u(x), v(x)) \\
v^{\prime}(x)=\varphi^{-}(x, u(x), v(x))
\end{array}\right.
\end{aligned}
$$

By the Lipschitz condition the interval problem and the ODEs (20), (21) have altogether two unique solutions locally. The above reasoning shows that any solution of the interval problem is a solution of the differential algebraic equation (17) and at its turn it is a solution for one of the ODE systems above. As a conclusion all the formulations are equivalent.

Remark 35. It is interesting to observe the meaning of the two equations in (17); The first equation (divide both sides by two) defines the midpoint of $y^{\prime}(x)$ to be the midpoint of $f(x, y)$ (and it finds the core of the solution) while the second equation finds the length of the solution as a function of time. Since we have locally two solutions we are able to track on the run the critical points. These are obtained as the points where the length of the solution is critical. In the crisp case, we will have $\varphi^{+}(x, u(x), v(x))-\varphi^{-}(x, u(x), v(x))=0$ so that $\left|u^{\prime}(x)-v^{\prime}(x)\right|=0$ and $u^{\prime}(x)=v^{\prime}(x)$ reducing (17) to the ordinary non interval case.

Equation (17) is a differential algebraic equation (DAE) in the implicit form, written in terms of $u(x), v(x)$ and their derivatives; it is not an ODE because it contains an absolute value and it is not possible to explicit the equations in terms of $u^{\prime}(x), v^{\prime}(x)$. We can obtain even more equivalent formulations as differential algebraic systems (see [2], [32], [10], [11] and [38] for general references and solution methods to DAEs). Indeed, we can write (17) as

$$
\begin{gathered}
\left\{\begin{array}{c}
u^{\prime}(x)+v^{\prime}(x)=\theta(x, u(x), v(x)) \\
\left|u^{\prime}(x)-v^{\prime}(x)\right|=\psi(x, u(x), v(x))
\end{array}\right. \\
\text { where }
\end{gathered}
$$

and observe that $\psi(x, u(x), v(x)) \geq 0$; then the second equation is equivalent to $\left[u^{\prime}(x)-v^{\prime}(x)\right]^{2}=\psi^{2}(x, u(x), v(x))$ so removing the non differentiability 
given by the absolute value. We then obtain the following DAE

$$
\left\{\begin{array}{c}
u^{\prime}(x)+v^{\prime}(x)=\theta(x, u(x), v(x)) \\
{\left[u^{\prime}(x)-v^{\prime}(x)\right]^{2}=\psi^{2}(x, u(x), v(x))}
\end{array}\right.
$$

and defining $w=u^{\prime}-v^{\prime}$ we obtain (after some algebra) an index-1 semi implicit DAE of the form

$$
\left\{\begin{array}{c}
u^{\prime}(x)=\frac{1}{2}(\theta(x, u(x), v(x))+w(x)) \\
v^{\prime}(x)=\frac{1}{2}(\theta(x, u(x), v(x))-w(x)) \\
{[w(x)]^{2}=\psi^{2}(x, u(x), v(x)) .}
\end{array}\right.
$$

By differentiating the third equation in (24) with respect to $x$ ( so $w^{\prime}=u^{\prime \prime}-v^{\prime \prime}$ and $2 w w^{\prime}=2\left(u^{\prime}-v^{\prime}\right)\left(u^{\prime \prime}-v^{\prime \prime}\right)=2 \psi\left[\psi_{x}+\psi_{u} u^{\prime}+\psi_{v} v^{\prime}\right]$ where $\psi_{x}, \psi_{u}$ and $\psi_{v}$ denote the partial derivatives of $\psi$ ) we finally obtain

$$
\begin{aligned}
& A(x, z) z^{\prime}=b(x, z) \\
& \text { with } \\
& A(x, z)=\left[\begin{array}{lll}
1 & 1 & 0 \\
1 & -1 & 0 \\
-\psi \psi_{u} & -\psi \psi_{v} & w
\end{array}\right], z=\left[\begin{array}{l}
u \\
v \\
w
\end{array}\right], b(x, z)=\left[\begin{array}{l}
\theta \\
w \\
\psi \psi_{x}
\end{array}\right] .
\end{aligned}
$$

and (consistent) initial conditions are

$$
\begin{aligned}
& u\left(x_{0}\right)=u_{0}, v\left(x_{0}\right)=v_{0}, \\
& w\left(x_{0}\right)=\left\{\begin{array}{c}
\varphi^{+}\left(x_{0}, u_{0}, v_{0}\right)-\varphi^{-}\left(x_{0}, u_{0}, v_{0}\right) \\
\text { or } \\
\varphi^{-}\left(x_{0}, u_{0}, v_{0}\right)-\varphi^{+}\left(x_{0}, u_{0}, v_{0}\right)
\end{array}\right.
\end{aligned}
$$

Matrix $A(x, z)$ has $\operatorname{det}(A(x, z))=2 w$ and is nonsingular if $w \neq 0$. If $w=0$ then $u^{\prime}=v^{\prime}$ and $u^{\prime}=\theta / 2, v^{\prime}=\theta / 2$ from the first equation in (25).

The formulation of IDE (14) in terms of DAE (24) or (25) can be useful to obtain a solution method. Observe first that the algebraic equation $w^{2}=$ $\psi^{2}$ in (24) can be solved for each $x$ by $w=\psi$ or by $w=-\psi$ producing, correspondingly, equations (21) or (20). To have a continuous right hand side in the differential equations of (24) the value of $w(x)$ can switch from $w=$ $\psi$ to $w=-\psi$ (or viceversa) only at points $\widehat{x}$ where $\psi(\widehat{x}, u(\widehat{x}), v(\widehat{x}))=0$, i.e. where $\varphi^{-}(\widehat{x}, u(\widehat{x}), v(\widehat{x}))=\varphi^{+}(\widehat{x}, u(\widehat{x}), v(\widehat{x}))$. At these switching points $\widehat{x}$, the solution(s) of (24) continues with two different branches, one obtained 
with $w(\widehat{x})=\psi(\widehat{x}, u(\widehat{x}), v(\widehat{x}))$, and one with $w(\widehat{x})=-\psi(\widehat{x}, u(\widehat{x}), v(\widehat{x}))$; in terms of functions $u(x)$ and $v(x)$, the two branches are in fact characterized by equations (10) and (9) respectively, i.e. by conditions $u^{\prime}(x) \geq v^{\prime}(x)$ and $u^{\prime}(x) \leq$ $v^{\prime}(x)$.

We illustrate the basic facts by the following example.

Example 36. The interval DE

$$
y^{\prime}=y \sin (x)
$$

can be written as (here $w(x)=u^{\prime}(x)-v^{\prime}(x)$ and $\left.k \in \mathbb{Z}\right)$

$$
\left\{\begin{array}{c}
u^{\prime}(x)+v^{\prime}(x)=(u(x)+v(x)) \sin (x) \\
|w(x)|=\left\{\begin{array}{l}
(v(x)-u(x)) \sin (x) \text { if } \quad x \in[2 k \pi,(2 k+1) \pi] \\
(u(x)-v(x)) \sin (x) \text { if } x \in[(2 k+1) \pi,(2 k+2) \pi] .
\end{array}\right.
\end{array}\right.
$$

For $x \in[2 k \pi,(2 k+1) \pi]$ the two equations (20)-(21), corresponding to $u^{\prime}(x) \geq$ $v^{\prime}(x)$ and $u^{\prime}(x) \leq v^{\prime}(x)$, respectively, are

$$
\left\{\begin{array} { l } 
{ u ^ { \prime } ( x ) + v ^ { \prime } ( x ) = ( u ( x ) + v ( x ) ) \operatorname { s i n } ( x ) } \\
{ u ^ { \prime } ( x ) - v ^ { \prime } ( x ) = ( v ( x ) - u ( x ) ) \operatorname { s i n } ( x ) }
\end{array} \text { i.e. } \left\{\begin{array}{l}
u^{\prime}(x)=v(x) \sin (x) \\
v^{\prime}(x)=u(x) \sin (x)
\end{array}\right.\right.
$$

and

$$
\left\{\begin{array} { l } 
{ u ^ { \prime } ( x ) + v ^ { \prime } ( x ) = ( u ( x ) + v ( x ) ) \operatorname { s i n } ( x ) } \\
{ u ^ { \prime } ( x ) - v ^ { \prime } ( x ) = ( u ( x ) - v ( x ) ) \operatorname { s i n } ( x ) }
\end{array} \text { i.e. } \left\{\begin{array}{l}
u^{\prime}(x)=u(x) \sin (x) \\
v^{\prime}(x)=v(x) \sin (x)
\end{array}\right.\right.
$$

For $x \in[(2 k+1) \pi,(2 k+2) \pi]$ the two equations, respectively, are

$$
\left\{\begin{array} { l } 
{ u ^ { \prime } ( x ) + v ^ { \prime } ( x ) = ( u ( x ) + v ( x ) ) \operatorname { s i n } ( x ) } \\
{ u ^ { \prime } ( x ) - v ^ { \prime } ( x ) = ( u ( x ) - v ( x ) ) \operatorname { s i n } ( x ) }
\end{array} \text { i.e. } \left\{\begin{array}{l}
u^{\prime}(x)=u(x) \sin (x) \\
v^{\prime}(x)=v(x) \sin (x)
\end{array}\right.\right.
$$

and

$$
\left\{\begin{array} { l } 
{ u ^ { \prime } ( x ) + v ^ { \prime } ( x ) = ( u ( x ) + v ( x ) ) \operatorname { s i n } ( x ) } \\
{ u ^ { \prime } ( x ) - v ^ { \prime } ( x ) = ( v ( x ) - u ( x ) ) \operatorname { s i n } ( x ) }
\end{array} \text { i.e. } \left\{\begin{array}{l}
u^{\prime}(x)=v(x) \sin (x) \\
v^{\prime}(x)=u(x) \sin (x)
\end{array} .\right.\right.
$$

So, in both cases, we have an alternation of two distinct ODEs.

It appears a first interpretation of the two solutions; note that $u(x)$ starts, at $x=x_{0}$, lower then $v(x)$ for interval initial condition $u\left(x_{0}\right) \leq v\left(x_{0}\right)$; equation 
(solution of) (20), characterized by the inequality $u^{\prime}(x) \geq v^{\prime}(x)$, is such that the distance between $u(x)$ and $v(x)$ will be reduced; equation (solution of) (21), characterized by the inequality $u^{\prime}(x) \leq v^{\prime}(x)$, is such that the distance between $u(x)$ and $v(x)$ will be increased. These cases correspond, as expected, to the two ways how interval uncertainty propagates in a dynamical system.

We end this section by shortly considering the case of a system of $n$ interval differential equations, to show that they can be handled in a similar way.

Let $f_{i}\left(x, y_{1}, \ldots, y_{n}\right)=\left[f_{i}^{-}\left(x, y_{1}, \ldots, y_{n}\right), f_{i}^{+}\left(x, y_{1}, \ldots, y_{n}\right)\right], i=1,2, \ldots, n$, be the interval valued functions at the right hand side of the system of IDEs

$$
\begin{aligned}
y_{i}^{\prime}(x) & =f_{i}\left(x, y_{1}(x), \ldots, y_{n}(x)\right) \text { with } x \in\left[x_{0}, X\right] \\
y_{i}\left(x_{0}\right) & =y_{i 0}=\left[u_{i 0}, v_{i 0}\right] \text { given at } x=x_{0}
\end{aligned}
$$

and suppose that each $y_{i}(x)$ is gH-differentiable, i.e. $y_{i}(x)=\left[u_{i}(x), v_{i}(x)\right]$ and

$$
\begin{aligned}
& y_{i}^{\prime}(x)=\left[\left(y_{i}^{\prime}(x)\right)^{-},\left(y_{i}^{\prime}(x)\right)^{+}\right], i=1,2, \ldots, n \\
& \quad \text { with } \\
& \left(y_{i}^{\prime}(x)\right)^{-}=\min \left\{u_{i}^{\prime}(x), v_{i}^{\prime}(x)\right\} \\
& \left(y_{i}^{\prime}(x)\right)^{+}=\max \left\{u_{i}^{\prime}(x), v_{i}^{\prime}(x)\right\} .
\end{aligned}
$$

Then, after defining $w_{i}=u_{i}^{\prime}-v_{i}^{\prime}$ we arrive at the DAE system

$$
\begin{aligned}
u_{i}^{\prime}-v_{i}^{\prime} & =w_{i} \\
u_{i}^{\prime}+v_{i}^{\prime} & =\theta_{i} \\
w_{i}^{2} & =\psi_{i}^{2} \\
\text { for } i & =1,2, \ldots, n
\end{aligned}
$$

where

$$
\begin{aligned}
\theta_{i}\left(x, u_{1}(x), v_{1}(x), \ldots, u_{n}(x), v_{n}(x)\right) & =\varphi_{i}^{+}\left(x, u_{1}(x), v_{1}(x), \ldots, u_{n}(x), v_{n}(x)\right) \\
& +\varphi_{i}^{-}\left(x, u_{1}(x), v_{1}(x), \ldots, u_{n}(x), v_{n}(x)\right) \\
\psi_{i}\left(x, u_{1}(x), v_{1}(x), \ldots, u_{n}(x), v_{n}(x)\right) & =\varphi_{i}^{+}\left(x, u_{1}(x), v_{1}(x), \ldots, u_{n}(x), v_{n}(x)\right) \\
& -\varphi_{i}^{-}\left(x, u_{1}(x), v_{1}(x), \ldots, u_{n}(x), v_{n}(x)\right)
\end{aligned}
$$

We obtain a system of $3 n$ differential algebraic equations and the switching can occur at points $\widehat{x}$ where $\psi_{i}\left(x, u_{1}(x), v_{1}(x), \ldots, u_{n}(x), v_{n}(x)\right)=0$ for at least one index $i \in\{1,2, \ldots, n\}$.

The topic of systems of interval differential equations, based on the above discussion, carries further problems that will need to be addressed in a future work. For example the possible switches between the differentiability cases are not easy to be identified and also, a related question is how the differentiability case that we chose for one of the unknowns influences the behavior of the other unknowns. 


\section{Solution methods for interval differential equations}

From the results and discussion of previous sections, the interval differential equation concept presented in this paper does not coincide with the concept of a differential inclusion. It is a different, new approach to model interval uncertainty in dynamical systems. It is related (as it is seen from the above theorems) to differential inclusions but we do have in our case more than one solution. The existence of several solutions can be an advantage when we search for solutions with specific properties like e.g., periodic, almost periodic, asymptotically stable, etc. Also, it can be very useful when we have unknown correlations between the parameters. In those situations, the uncertainty about the correlation introduces supplementary uncertainty in our system, so the existence of several solutions appears to be natural in this case.

The above characterization theorems, together with the existence results, lead easily to a numerical algorithm to solve interval differential equations.

\subsection{General description of the solution methods}

First, let us remark that a switch between the cases (i) and (ii) of gH-differentiability is possible if and only if $y^{\prime}(x)$ is a singleton as in fact possible switch-points are l-critical points, i.e., critical points of the length of $y(x)$. Let us remark that if at a point $y\left(x_{0}\right)$ is a singleton for some $x_{0}$, then this point is enforcing a switch to the case (i), because, according to the existence result in Theorem 31, the second solution does not exist in this case. All other critical points make possible a switch from (i) to (ii) case or viceversa, but they do not enforce a switch, so at each l-critical point $x_{0}$ with $y\left(x_{0}\right)$ nontrivial interval, two new local solutions arise. One is (i)-differentiable, the other is (ii)-differentiable.

These remarks, together with the characterization Theorem 32, lead to the following general approach to numerically solve interval differential equations. We solve basically ODEs (9) and (10) on subintervals of the time domain having the initial value $y_{0}$ updated at all the possible critical points. In this way on a bounded time interval we obtain a finite number of solutions. For the solution of (9) and (10) by the characterization theorem above, any efficient numerical method for ODEs can be used. This is an advantage of the method presented here, as we do not need to reinvent numerical methods for interval differential equations. Instead we can use the classical ones on the ODE translations of the interval differential equations.

This algorithm generates a tree structure for the solutions of the IDE. All the nodes of the tree will be critical points except the terminal nodes. All the branches represent local solutions of (i) or (ii) kind between two nodes. This 
is illustrated in Figure 3.

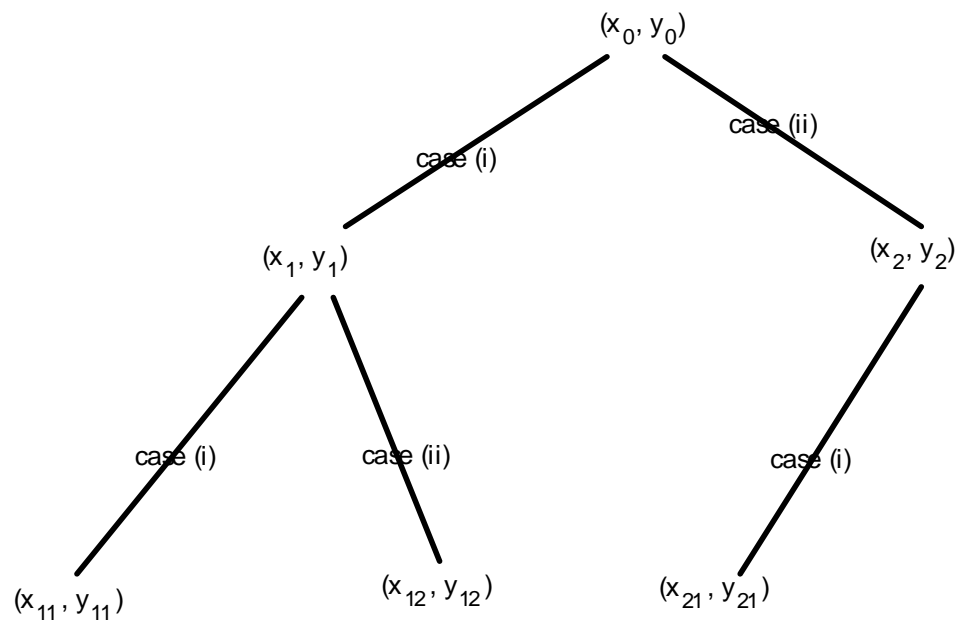

Figure 3. The structure of the tree of local solutions.

Each node represents an l-critical point with a switching and each branch corresponds to one of the cases (i) or (ii) of differentiability.

Our algorithm which generates all the solutions on a bounded interval is as follows.

\section{Algorithm 1. (Find solutions of an interval differential equation - IDE)}

Let us consider $y_{0}$ be any interval.

- Step 1. If $y_{0}$ is a singleton then we solve (9) and we obtain solution $y_{1}$.

- Step 2. Else we solve both (9) and (10) and we obtain solutions $y_{1}, y_{2}$.

- Step 3. Let $x_{1}=\inf \left\{x>x_{0}: y_{1}^{\prime}(x) \in \mathbb{R}\right\}$ and $x_{2}=\inf \left\{x>x_{0}: y_{2}^{\prime}(x) \in \mathbb{R}\right.$ or $\left.y_{2}(x) \in \mathbb{R}\right\}$ be the nearest critical points or singleton values. (Let us remark here that since the algorithm applies to general interval-valued functions, it is possible that $y_{2}(x) \in \mathbb{R}$ however $\left.y_{2}^{\prime}(x) \notin \mathbb{R}\right)$

- Step 4. We insert the solutions which are found in a tree structure: $y_{1}$ in the left branch and $y_{2}$ in the right branch (the root will be simply $\left(x_{0}, y_{0}\right)$ ).

- Step 5. If $x_{1}<X$ (a preset maximum value for $x$ ) then let $x_{0}=x_{1}$ and $y_{0}=y_{1}\left(x_{1}\right)$ and go to step 1 .

- Step 6. If $x_{2}<X$ then let $x_{0}=x_{2}$ and $y_{0}=y_{2}\left(x_{2}\right)$ and go to step 1 .

- Step 7. Else Return.

- Step 8. Using a standard backtracking algorithm we generate all the solutions from the tree structure generated above. 
The presented algorithm will generate a finite number of solutions on the interval $\left[x_{0}, X\right]$ provided that there are a finite number of critical points. Later we can extract those solutions which are closely reflecting the phenomenon which we have to model.

When $f(x, y)$ is the interval extension of a continuous function $f(x, p), p \in \mathbb{R}$ and if we are interested in finding the attainable set for the differential inclusions $y^{\prime}=f(x, y), y\left(x_{0}\right) \in y_{0}, x \in\left[x_{0}, X\right]$, then we have a simpler algorithm based on the characterization Theorem 32 and the results shown in Theorem 33 .

Let us consider $y_{0}$ be any interval. Let $f(x, y)$ be the interval extension of a real function $f(x, p)$. We use the same notation for both functions, and from the context we can identify them.

\section{Algorithm 2. (Find the attainable set of a differential inclusion)}

- Step 1. We find the points where the real function $f$ changes its monotonicity w.r.t. $p$ (if $f$ is differentiable then we solve $\frac{\partial f}{\partial p}=0$ and we find the critical points where monotonicity w.r.t. the second variable is changed). Let $\left(x_{1}, y_{1}\right)$ be a critical point such that in $] x_{0}, x_{1}$ [ there are no other critical points.

- Step 2. If $f$ is increasing w.r.t. $p$ on $\left[x_{0}, x_{1}\right] \times\left[y_{0}, y_{1}\right]$ we solve $(9)$

- Step 4. Else if $f$ is decreasing w.r.t. $p$ on $\left[x_{0}, x_{1}\right] \times\left[y_{0}, y_{1}\right]$ we solve $(10)$

- Step 5. If $x_{1}<X$ then let $x_{0}=x_{1}$ and $y_{0}=y\left(x_{1}\right)$ and go to step 2 .

- Else Stop.

This algorithm leads to the unique solution (attainable set) of the differential inclusion $y^{\prime}=f(x, y), y(0) \in y_{0}$.

It is easy to see that the proposed methods are very efficient from the numerical point of view, since for the local solutions we can use any standard algorithm.

\subsection{Examples}

The above algorithms were implemented in MATLAB. We have used MATLAB's standard ODE solver ode45, which is based on a Runge-Kutta $(4,5)$ formula, the Dormand-Prince pair. Surely, any other solver could be used. Let us remark here that the critical points were in all the cases a priori determined. The critical points cannot be easily found on the run. The problem is that if we detect a critical point, due to the machine precision the algorithm finds further points which are close to the correct critical point as critical ones. Also, if we have a solution in the case (ii) with length decreasing asymptotically to zero, due to the machine precision the program detects them as critical points. 
We will start with a simple example, which is easy to be solved analytically and we can compare the analytical solution to the numerical solution.

Example 37. Let us consider the interval differential equation

$$
\left\{\begin{array}{c}
y^{\prime}=-y+[1,2] x \\
y(0)=[0,1]
\end{array} x \in[0,4] .\right.
$$

We denote $y=[u, v]$, where $u, v$ are real-valued functions. The systems (9) and (10) are respectively

$$
\begin{gathered}
\left\{\begin{array}{c}
u^{\prime}=-v+x \\
v^{\prime}=-u+2 x \\
u(0)=0 \\
v(0)=1
\end{array}\right. \\
\left\{\begin{array}{c}
u^{\prime}=-u+2 x \\
v^{\prime}=-v+x \\
u(0)=0 \\
v(0)=1
\end{array}\right.
\end{gathered}
$$

This equation (29) has exactly two solutions. One of them starts with the case (i) of differentiability

$$
y_{1}(x)=\left[2 x-e^{x}+2 e^{-x}-1, x+e^{x}+2 e^{-x}-2\right]
$$

and there are no critical points in the trajectory.

If we start with case (ii) then we have a critical point of type II at $x=1$ (i.e., $y(1)$ is a singleton). In this case we have to switch to case (i) of differentiability since the (ii)-differentiable solution does not exist. We obtain

$$
\begin{aligned}
& u(x)=\left\{\begin{array}{cc}
2 x+2 e^{-x}-2 \quad \text { if } 0 \leq x \leq 1 \\
2 x-e^{x-1}+2 e^{-x}-1 \text { if } \quad 1 \leq x
\end{array}\right. \\
& v(x)=\left\{\begin{array}{cc}
x+2 e^{-x}-1 \quad \text { if } 0 \leq x \leq 1 \\
x+e^{x-1}+2 e^{-x}-2 \text { if } \quad 1 \leq x
\end{array}\right.
\end{aligned}
$$

The analytic solution and the numerical solutions obtained by the proposed algorithms are shown in Figs 4. and 5. respectively. We can see that the proposed method is very accurate. The error is controlled by the precision of 
ode 45 algorithm in MATLAB, and it is less than $10^{-6}$.

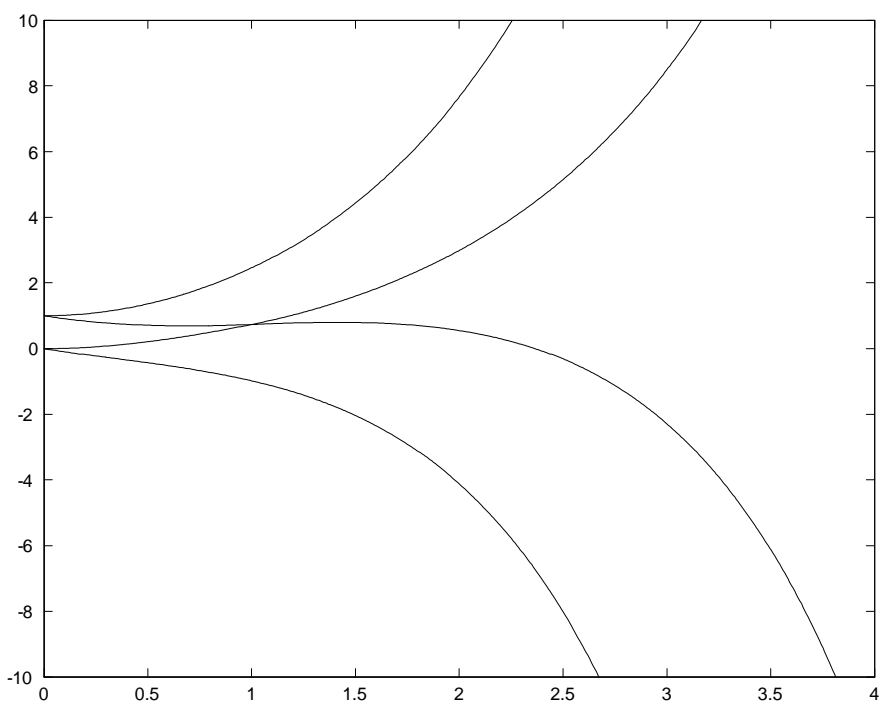

Figure 4. Analytic (exact) solutions of the IDE (29)

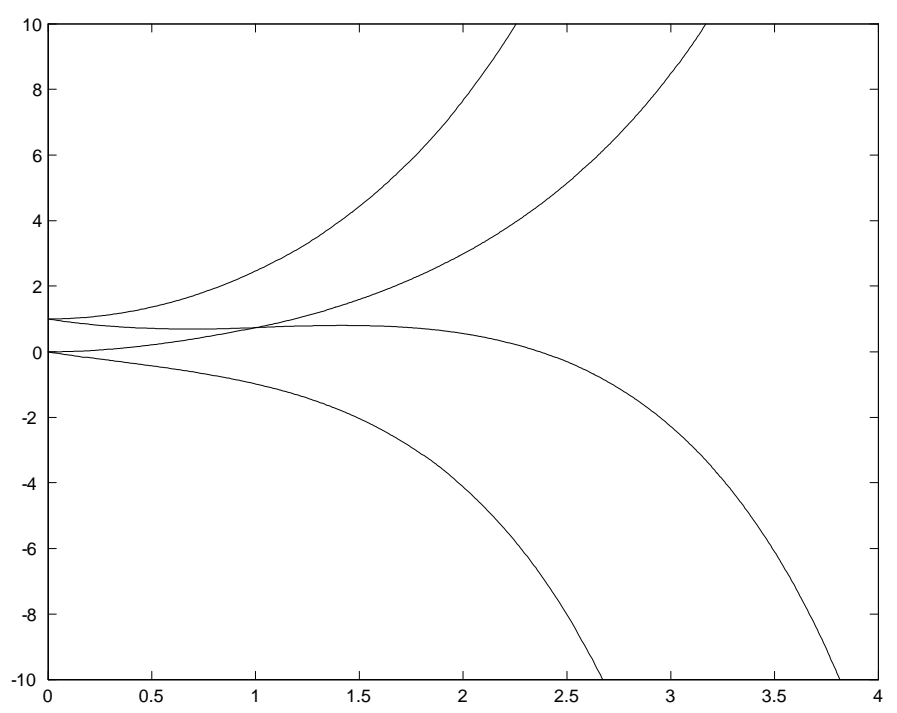

Figure 5. Numerical solutions of (29) by the proposed Algorithm 1.

Example 38. Consider the interval problem

$$
\left\{\begin{array}{c}
y^{\prime}=-y+[1,2] \sin x, x \in[0,4] \\
y(0)=[1,3] .
\end{array}\right.
$$

The numerical solutions are presented in Figure 6. In this case the switching point of the second type is determined numerically. We can see that there are 
no other critical points.

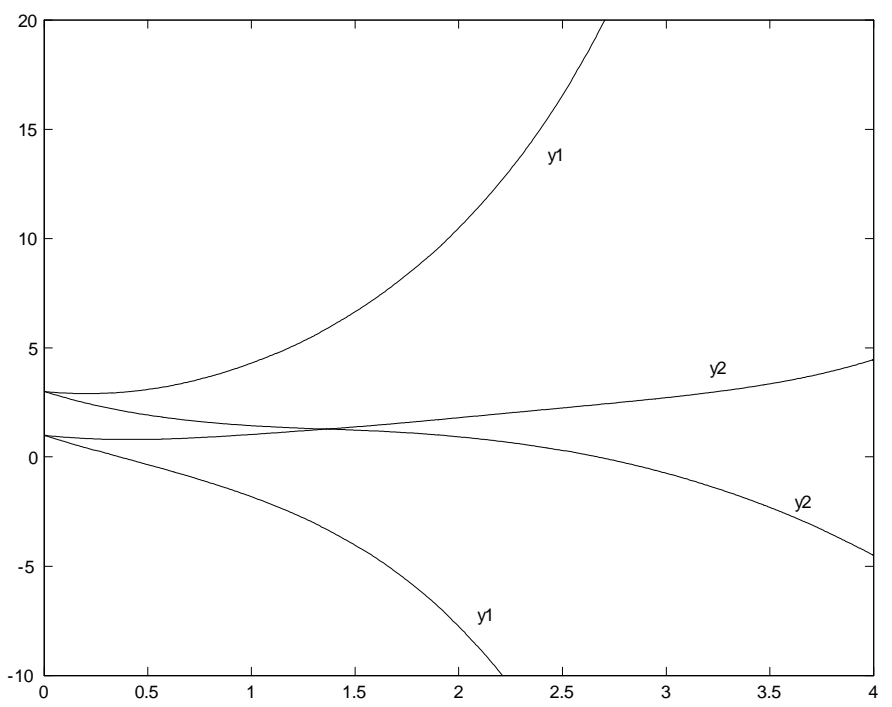

Figure 6. Solutions af IDE (32) by Algorithm 1.

There is a critical point (switching) between 1 and 1.5

Example 39. The structure of solution of an interval DE can be well illustrated on the problem

$$
\left\{\begin{array}{c}
y^{\prime}=y \sin x, x \in[0,6] \\
y(0)=[1,2]
\end{array}\right.
$$

According to the characterization theorems it can be written as

$$
\left\{\begin{array}{l}
u^{\prime}(x)=u(x) \sin (x) \\
v^{\prime}(x)=v(x) \sin (x)
\end{array}\right.
$$

or

$$
\left\{\begin{array}{l}
u^{\prime}(x)=v(x) \sin (x) \\
v^{\prime}(x)=u(x) \sin (x)
\end{array} .\right.
$$

The l-critical points are at $x=k \pi, k \in \mathbb{Z}$. If we solve the problem in the interval $[0, X]$ with $X<2 \pi$ we have only one critical point in the interval. 
Then we may have four solutions, as illustrated in Figure 7.

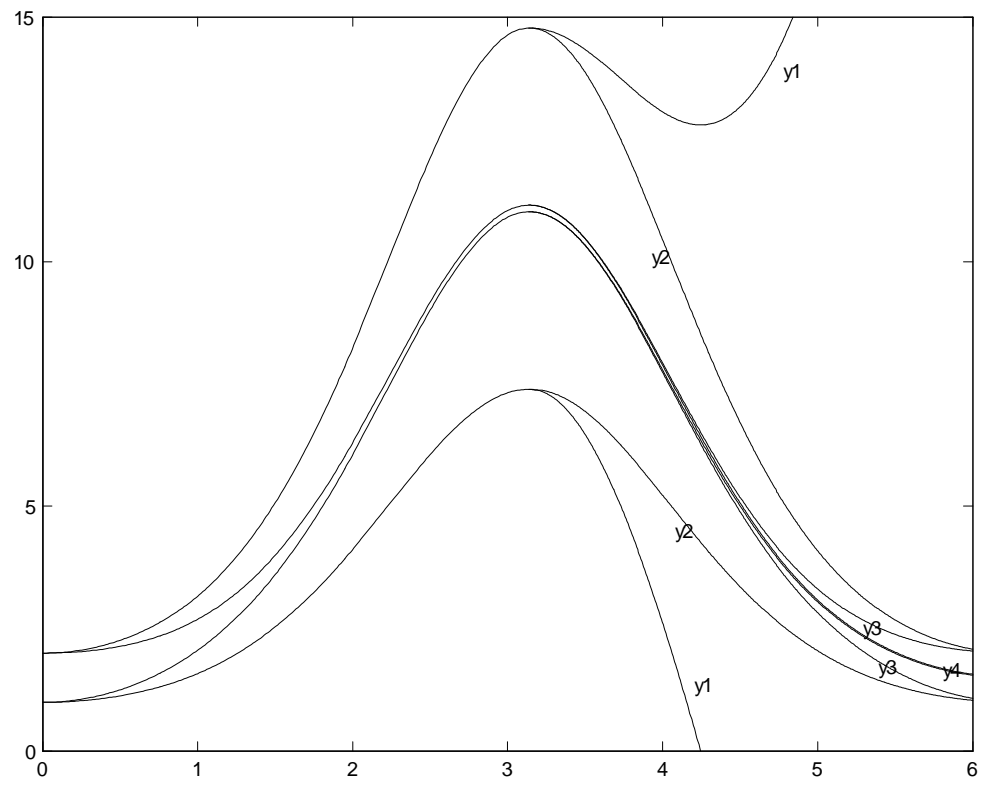

Figure 7 . The four solutions of IDE (33) on the interval $[0,6]$

If we consider time intervals containing more critical points, we may have more solutions. In Figure 8. we illustrate the solutions found by Algorithm 1 to IDE (33) for $x \in[0,11]$.

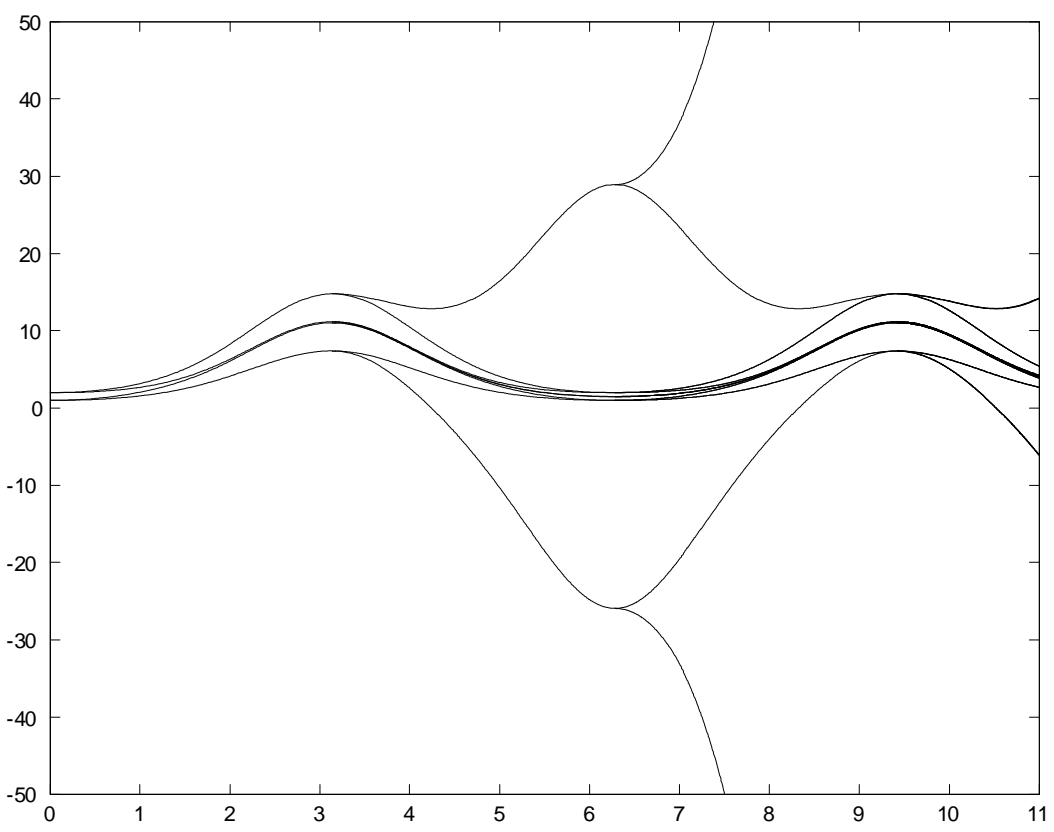

Figure 8. Existence of several solutions for IDE (33) on $[0,11]$

Example 40. Regarding the second algorithm for solving differential inclu- 
sions when the function $f(x, y)$ is the interval extension of a crisp function we present the following problem

$$
\left\{\begin{array}{c}
y^{\prime}=y \sin x, x \in[0,6] \\
y(0) \in[1,2]
\end{array}\right.
$$

We observe that the function $y \sin x$ changes monotony at the points $k \pi, k \in \mathbb{Z}$. Also, we observe that in the interval $[0, \pi]$ it is increasing w.r.t. $y$. Using the Algorithm 2 proposed for differential inclusions we obtain the solution presented in Figure 9.

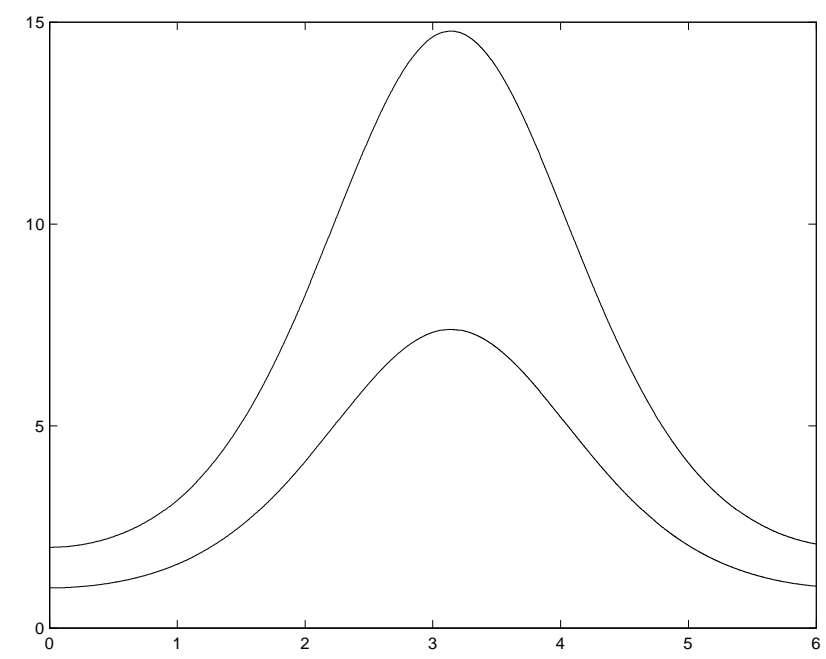

Figure 9. Solution of differential inclusion (34) using the proposed Algorithm 2

\section{Conclusions}

Interval differential equations with a generalized Hukuhara type differentiability are studied both from theoretical and practical points of view. We have studied the generalized Hukuhara difference and differentiability for intervals. We obtained an existence theorem and uniqueness of two solutions. Also, a characterization by ODEs is proposed together with a numerical procedure to solve interval differential equations.

We obtain an interesting connection between interval differential equations and differential algebraic systems. We plan to further exploit this connection twofold. First direction would be to use DAEs for solving IDEs. But also possibly, it is possible to use IDEs to solve some DAEs, since their theory is also not very well understood. 
Also, let us remark here that it is easy to extend the definition of the $\mathrm{gH}$ differentiability to define higher order gH-derivatives and partial gH-derivatives. So, higher order interval differential equations, and interval partial differential equations by means of the gH-derivative are subjects for further study. These could be of interest since existing approaches to interval PDEs namely interval finite element methods carry in many situations the problem of overestimation

of uncertainty (see [26]). How parameter uncertainty propagates in systems described by PDEs is a topic of interest in many safety-related applications.

\section{References}

[1] R.P. Agarwal, D. O'Regan, Existence for set differential equations via multivalued operator equations. Differential equations and applications. Vol. 5, 1-5, Nova Sci. Publ., New York, 2007.

[2] U.M. Ascher, L.R. Petzold, Computer Methods for ordinary differential equations and differential algebraic equations, SIAM, 1999.

[3] J.P. Aubin, A. Cellina, Differential Inclusions, Springer-Verlag, Berlin, Heidelberg, New York, Tokyo, 1984.

[4] R. Baker Kearfott, V. Kreinovich (Eds.) Applications of interval computations, Kluwer Academic Publishers, 1996.

[5] B. Bede, Note on "Numerical solutions of fuzzy differential equations by predictor-corrector method", Information Sciences, 178(2008) 1917-1922.

[6] B. Bede, S.G. Gal, Generalizations of the differentiability of fuzzy-numbervalued functions with applications to fuzzy differential equations, Fuzzy Sets and Systems, 151(2005), 581-599.

[7] Y. Chalco-Cano, H. Román-Flores, On new solutions of fuzzy differential equations Chaos, Solitons \& Fractals, 38(2008), 112-119.

[8] T. Gnana Bhaskar, V. Lakshmikantham, Set differential equations and Flow invariance, J. Appl. Anal. 82 (2) (2003) 357-368.

[9] F.S. de Blasi, V. Lakshmikantham, T. G. Bhaskar, An existence theorem for set differential inclusions in a semilinear metric space. Control Cybernet. 36 (2007), no. $3,571-582$.

[10] K.E. Brenan, S.L. Campbell, L.R. Petzold, Numerical Solution of initial-value problems in differential algebraic equations, North Holland, New York, 1989; republished by SIAM, 1996.

[11] S.L. Campbell, C.W. Gear, The index of general nonlinear DAEs, Numerische Mathematik, 72(1995), 173-196.

[12] K. Deimling, Multivalued Differential Equations. W. De Gruyter, Berlin, 1992. 
[13] P. Diamond, Stability and periodicity in fuzzy differential equations, IEEE Trans. Fuzzy Systems 8(2000) 583-590.

[14] P. Diamond, P. Kloeden, Metric Spaces of Fuzzy Sets, World Scientific, New Jersey, 1994.

[15] M. Feckan, Chaos in ordinary differential equations with perturbations: Applications to dry friction problems, Nonlinear Analysis, Theory, Methods \& Applications, 30(1997) 1355-1364.

[16] Gotz Alefeld, Gunter Mayer, Interval analysis: theory and applications, Journal of Computational and Applied Mathematics 121 (2000) 421-464.

[17] T. Johnson, W. Tucker, Rigorous parameter reconstruction for differential equations with noisy data, Automatica, in press.

[18] E. Hüllermeier, An approach to modelling and simulation of uncertain dynamical systems, Int. J. Uncertainty, Fuzziness and Knowledge-Based Systems, 5(1997), 117-137.

[19] M. Hukuhara, Intégration des applications mesurables dont la valeur est un compact convex, Funkcial. Ekvac. 10 (1967) 205-229.

[20] O. Kaleva, Fuzzy differential equations, Fuzzy Sets and Systems, 24(1987), 301317.

[21] P.E. Kloeden, Remarks on Peano theorem for fuzzy differential equations, Fuzzy Sets and Systems, 44(1991), 161-163.

[22] M. Kunze, Non-Smooth Dynamical Systems, Springer, 2000.

[23] V. Lakshmikantham, A.A. Tolstonogov, Existence and interrelation between set and fuzzy differential equations, Nonlinear Anal. 55 (2003), no. 3, 255-268.

[24] V. Lakshmikantham, R. Mohapatra, Theory of Fuzzy Differential Equations and Inclusions, Taylor \&Francis, London, 2003.

[25] K. Makino, M. Berz, Efficient control of the dependency problem based on Taylor model methods, Reliable Computing, 5, No. 1 (1999), 3-12.D.

[26] D. Moens and D. Vandepitte. A survey of non-probabilistic uncertainty treatment in finite element analysis. Computer Methods in Applied Mechanics and Engineering, 194(14-16):1527-1555, 2005.

[27] R.E. Moore, Interval Analysis, Prentice-Hall, Englewood Cliffs, NJ, 1966.

[28] N. S. Nedialkov, K. R. Jackson, J. D. Pryce, An effective high order interval method for validating existence and uniqueness of the solution of an IVP for an ODE, Reliable Computing, 7, No. 6 (2001), 449-465.

[29] A. Neumaier, Rigorous sensitivity analysis for parameter dependent systems of equations, J. of Math. Anal. and Appl., 114 (1989), 16-25.

[30] A. Neumaier, Interval Methods for Systems of Equations, Cambridge University Press (1990). 
[31] J. J. Nieto, R. Rodríguez-López, Euler polygonal method for metric dynamical systems, Information Sciences, 177(2007), 4256-4270.

[32] L. Petzold, Differential algebraic equations are not ODEs, SIAM Journal on Scientific and Statistical Computing, 3(1982), 367-384.

[33] M. Puri, D. Ralescu, Differentials of fuzzy functions, J. Math. Anal. Appl., 91(1983), 552-558.

[34] R. Rodríguez-López, Comparison results for fuzzy differential equations, Information Sciences, 178(2008), 1756-1779.

[35] S. Seikkala, On the fuzzy initial value problem, Fuzzy Sets and Systems, 24(1987), 319-330.

[36] W. Sextro, Dynamical contact problems with friction, Models, Methods, Experiments and Applications, Springer, 2002.

[37] I. Skalna, M.V. Rama Rao, A. Pownuk, Systems of fuzzy equations in structural mechanics, Journal of Computational and Applied Mathematics, 218(2008), 149-156.

[38] P. Rabier, W. Rheinboldt, Theoretical and numerical analysis of differential algebraic equations, in P.G. Ciarlet, J.L.Lions (eds) Handbook of Numerical Analysis, vol. VIII, Elsevier, 2002.

[39] L. Stefanini, L. Sorini, M. L. Guerra, Parametric representation of fuzzy numbers and application to fuzzy calculus, Fuzzy Sets and Systems 157 (2006) $2423-2455$.

[40] L. Stefanini, A generalization of Hukuhara difference for interval and fuzzy arithmetic, submitted for publication, 2008. Working Papers 0801, University of Urbino Carlo Bo, Available online on the RePEC repository, ideas.repec.org (http://ideas.repec.org/f/pst233.html).

[41] L. Stefanini, L. Sorini, M. L. Guerra, Fuzzy numbers and Fuzzy Arithmetic, Chapter 12 in W. Pedrycz, A. Skowron, V. Kreinovich (eds), Handbook of Granular Computing, John Wiley \& Sons, Ltd, 2008.

[42] Congxin Wu, Shiji Song, E. Stanley Lee, Approximate solutions, existence and uniqueness of the Cauchy problem of fuzzy differential equations, J. Math. Anal. Appl., 202(1996), 629-644.

[43] L.A. Zadeh, Fuzzy Sets, Inform. and Control, 8(1965), 338-353. 